\title{
Deuterium Labeling Studies Establishing Stereochemistry at the Oxypalladation Step in Wacker-Type Oxidative Cyclization of an o-Allylphenol
} Tamio Hayashi,* Kaori Yamasaki, Michihiro Mimura, and Yasuhiro Uozumi

\author{
Department of Chemistry, Graduate School of Science, Kyoto University, \\ Sakyo, Kyoto 606-8502, Japan
}

\section{Supporting Information}

General. NMR spectra were recorded on a JEOL JNM LA500 spectrometer (500 MHz for ${ }^{1} \mathrm{H}, 76.5 \mathrm{MHz}$ for ${ }^{2} \mathrm{H}$, and $125 \mathrm{MHz}$ for ${ }^{13} \mathrm{C}$ ) in $\mathrm{CDCl}_{3}$. Chemical shifts are reported in $\delta$ ppm referenced to an internal $\mathrm{SiMe}_{4}$ standard for ${ }^{1} \mathrm{H}$ NMR. Chemical shifts for deuterium are reported in $\delta \mathrm{ppm}$ referenced to residual $\mathrm{CDCl}_{3}$ in $\mathrm{CHCl}_{3}$ as an internal standard. Residual chloroform $\left(\delta 77.0\right.$ for ${ }^{13} \mathrm{C}$ ) was used as internal reference for ${ }^{13} \mathrm{C}$ NMR. Detailed ${ }^{1} \mathrm{H}$ assignments were performed by $\mathrm{H}-\mathrm{H}$ cosy experiments. Air- and moisture-sensitive reactions were performed under usual inert atmosphere techniques.

Materials. Tetrahydrofuran and diethyl ether were distilled from benzophenone-ketyl under nitrogen prior to use. Dichloromethane was distilled from $\mathrm{CaH}_{2}$ under nitrogen. $\mathrm{Pd}(\mathrm{MeCN})_{4}\left(\mathrm{BF}_{4}\right)_{2}$ was purchased from Aldrich Chemical Co. Ltd., and used as received. $(S, S)-2,2^{\prime}$-Bis[4-(alkyl)oxazolyl]-1,1'-binaphthyls $((S, S) \text {-ip-boxax })^{1}$ and [(3,2,10- $\eta^{3}$-pinene)$\mathrm{PdOAc}]_{2}^{2}$ were prepared according to the reported procedures.

Palladium-Catalyzed Oxidative Cyclization of 1 in the Presence of $\operatorname{Pd}(\mathrm{II}) /(\boldsymbol{S}, \boldsymbol{S})$-ip-boxax Catalyst and $\boldsymbol{p}$-Benzoquinone. ${ }^{1}$ To a solution of $8.9 \mathrm{mg}$ (20 $\mu \mathrm{mol})$ of $\mathrm{Pd}(\mathrm{MeCN})_{4}\left(\mathrm{BF}_{4}\right)_{2}$ in acetonitrile $(0.2 \mathrm{~mL})$ was added $(S, S)$-ip-boxax $(19 \mathrm{mg}, 40$ $\mu \mathrm{mol})$ in $\mathrm{CH}_{2} \mathrm{Cl}_{2}(0.2 \mathrm{~mL})$ at room temperature. The mixture was stirred at room temperature for $3 \mathrm{~min}$ and concentrated in vacuo to give orange solid. To the orange solid was added $p$ benzoquinone $(0.17 \mathrm{~g}, 1.6 \mathrm{mmol}), \mathrm{MeOH}(0.3 \mathrm{~mL})$ and a solution of 3-(2- 
hydroxyphenyl)cyclohexene (1) $(69.6 \mathrm{mg}, 0.40 \mathrm{mmol})$ in $\mathrm{MeOH}(0.5 \mathrm{~mL})$. The reaction mixture was stirred at $40{ }^{\circ} \mathrm{C}$ for $4 \mathrm{~h}$ and then concentrated in vacuo. The residue was chromatographed on silica gel $($ EtOAc/n-hexane $=1 / 10)$ to give $54.1 \mathrm{mg}(78 \%)$ of the Wackertype cyclization product which consists of four regioisomers $\mathbf{2}, \mathbf{3}, \mathbf{4}$, and $\mathbf{5}$ in a ratio of $33 / 30 / 26 / 11$. The benzofuran 5 was isolated pure by preparative thin-layer chromatography on silica gel $($ EtOAc/n-hexane $=1 / 10)$. The remaining mixture of $\mathbf{2}, \mathbf{3}$, and $\mathbf{4}$ was separated by repeated preparative thin-layer chromatography $($ EtOAc/n-hexane $=1 / 30)$ into two parts, one mainly containing $\mathbf{2}$ and $\mathbf{3}$ and the other mainly containing 4. Figure 1 shows ${ }^{1} \mathrm{H}$ NMR spectra for $\mathbf{2}$ and 3, and that for 4 . Figure 2 shows assignment of ${ }^{1} \mathrm{H}$ NMR signals for $2, \mathbf{3}, \mathbf{4}$, and 5.

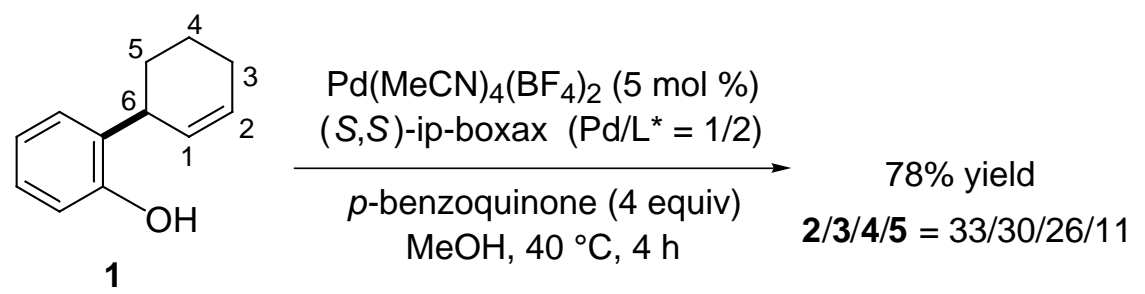<smiles>C1=CCC2c3ccccc3OC2[CH+]1</smiles>

2<smiles>C1=CCC2c3ccccc3OC2C1</smiles>

3<smiles>C1=CC2c3ccccc3OC2CC1</smiles>

4<smiles>c1ccc2c3c(oc2c1)CCCC3</smiles>

5

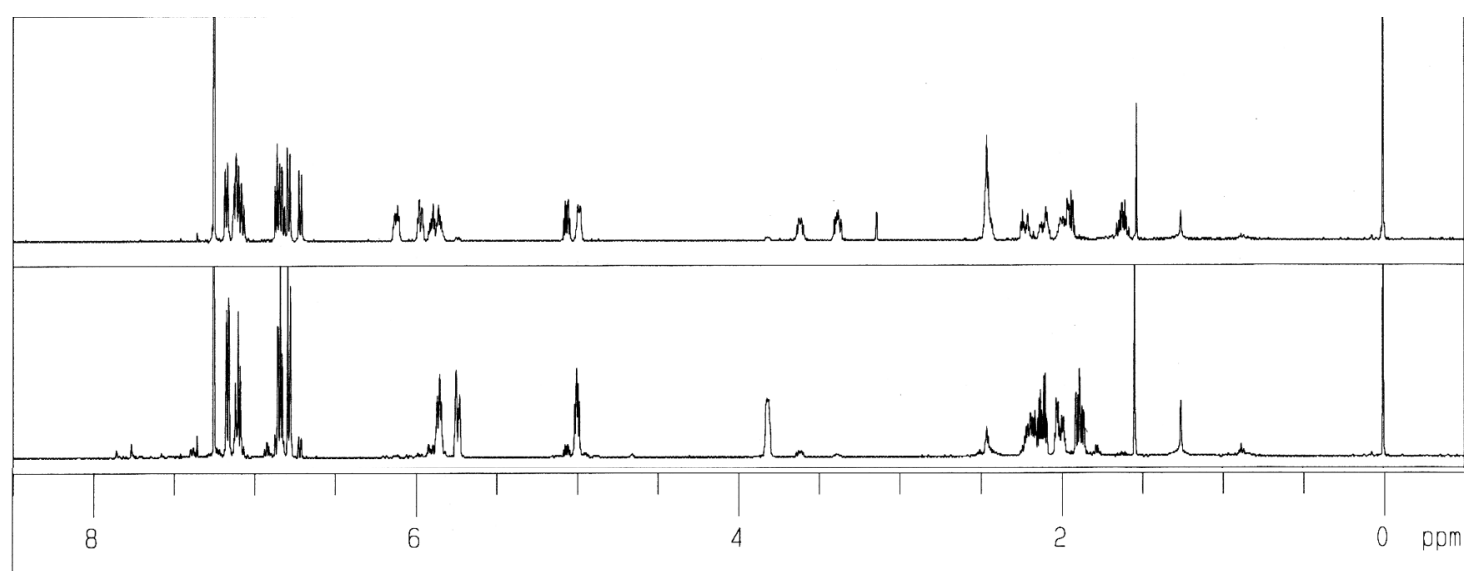

Figure 1. ${ }^{1} \mathrm{H}$ NMR chart for $\mathbf{2}+\mathbf{3}$ (upper) and $\mathbf{4}$ (lower) 
2: ${ }^{1} \mathrm{H} \mathrm{NMR}\left(\mathrm{CDCl}_{3}\right) \delta 1.62(\mathrm{dtd}, J=15.0,9.8,5.0 \mathrm{~Hz}, 1 \mathrm{H}), 1.95(\mathrm{dq}, J=15.0,5.0$ $\mathrm{Hz}, 1 \mathrm{H}), 1.94-2.02(\mathrm{~m}, 1 \mathrm{H}), 2.11$ (br dq, $J=18.2,5.0 \mathrm{~Hz}, 1 \mathrm{H}), 3.39$ (ddd, $J=9.8,8.3,4.8$ $\mathrm{Hz}, 1 \mathrm{H}), 4.99$ (br d, $J=8.3 \mathrm{~Hz}, 1 \mathrm{H}), 5.98$ (ddt, $J=10.1,3.7,1.9 \mathrm{~Hz}, 1 \mathrm{H}), 6.12$ (ddd, $J=$ 10.1, 5.0, 3.4 Hz, 1H), 6.79 (br d, $J=8.0 \mathrm{~Hz}, 1 \mathrm{H}), 6.86$ (br t, $J=7.5 \mathrm{~Hz}, 1 \mathrm{H}$ ), 7.12 (br t, $J$ $=8.0 \mathrm{~Hz}, 1 \mathrm{H}), 7.18(\mathrm{brd}, J=7.5 \mathrm{~Hz}, 1 \mathrm{H}) ;{ }^{13} \mathrm{C} \mathrm{NMR}\left(\mathrm{CDCl}_{3}\right) \delta 22.73,25.22,39.98$, 78.60, 109.87, 120.36, 123.95, 124.40, 128.04, 131.44, 133.34, 158.99. 3: ${ }^{1} \mathrm{H}$ NMR $\left(\mathrm{CDCl}_{3}\right) \delta 2.23$ (br dt, $\left.J=15.8,5.0 \mathrm{~Hz}, 1 \mathrm{H}\right), 2.42-2.47$ (br m, 1H), 2.42-2.49 (m, 1H), 2.44-2.49 (br m, 1H), 3.62 (ddd, $J=9.3,6.9,5.0 \mathrm{~Hz}, 1 \mathrm{H}), 5.07$ (dt, $J=9.3,5.0 \mathrm{~Hz}, 1 \mathrm{H})$, 5.86 (br dt, $J=9.6,4.5 \mathrm{~Hz}, 1 \mathrm{H}$ ), 5.90 (br dt, $J=9.6,4.5 \mathrm{~Hz}, 1 \mathrm{H}$ ), 6.72 (br d, $J=8.0 \mathrm{~Hz}$, 1H), 6.83 (br t, $J=7.5 \mathrm{~Hz}, 1 \mathrm{H}), 7.09$ (br t, $J=8.0 \mathrm{~Hz}, 1 \mathrm{H}$ ), 7.12 (br d, $J=7.5 \mathrm{~Hz}, 1 \mathrm{H}$ ); ${ }^{13} \mathrm{C} \mathrm{NMR}\left(\mathrm{CDCl}_{3}\right) \delta 28.01,28.33,40.03,82.00,108.99,120.26,124.12,125.81,128.37$, 129.50, 131.73, 159.89. 4: ${ }^{1} \mathrm{H}$ NMR $\left(\mathrm{CDCl}_{3}\right) \delta 1.88(\mathrm{dddd}, J=13.5,9.7,5.0,3.4 \mathrm{~Hz}$, $1 \mathrm{H}), 2.01$ (br dq, $J=17.1,5.0 \mathrm{~Hz}, 1 \mathrm{H}), 2.12(\mathrm{dq}, J=13.5,5.0 \mathrm{~Hz}, 1 \mathrm{H}), 2.18$ (dddq, $J=$ 17.1, 9.7, 5.0, $2.4 \mathrm{~Hz}, 1 \mathrm{H}), 3.82$ (br d, $J=7.5 \mathrm{~Hz}, 1 \mathrm{H}), 5.00$ (ddd, $J=7.8,5.0,3.4 \mathrm{~Hz}$, 1H), 5.74 (dquint, $J=10.0,2.4 \mathrm{~Hz}, 1 \mathrm{H}$ ), 5.86 (dddd, $J=10.0,5.0,3.1,1.9 \mathrm{~Hz}, 1 \mathrm{H}$ ), 6.79 $(\mathrm{dt}, J=8.0,0.5 \mathrm{~Hz}, 1 \mathrm{H}), 6.84(\mathrm{td}, J=7.5,1.0 \mathrm{~Hz}, 1 \mathrm{H}), 7.11(\mathrm{br} \mathrm{t}, J=8.0 \mathrm{~Hz}, 1 \mathrm{H}), 7.17$ (br d, $J=7.5 \mathrm{~Hz}, 1 \mathrm{H}) ;{ }^{13} \mathrm{C} \mathrm{NMR}\left(\mathrm{CDCl}_{3}\right) \delta 19.60,25.03,41.16,81.31,109.89,120.46$, 124.29, 126.27, 127.73, 128.06, 131.12, 159.21. 5: ${ }^{1} \mathrm{H}$ NMR $\left(\mathrm{CDCl}_{3}\right) \delta$ 1.82-1.88 (m, 2H), 1.91-1.97 (m, 2H), $2.63(\mathrm{tt}, J=6.1,1.9 \mathrm{~Hz}, 2 \mathrm{H}), 2.74(\mathrm{tt}, J=6.1,1.9 \mathrm{~Hz}, 2 \mathrm{H}), 7.19$ $(\mathrm{d}, J=7.1 \mathrm{~Hz}, 1 \mathrm{H}), 7.19(\mathrm{t}, J=5.5 \mathrm{~Hz}, 1 \mathrm{H}), 7.38-7.42(\mathrm{~m}, 2 \mathrm{H}) ;{ }^{13} \mathrm{C} \mathrm{NMR}\left(\mathrm{CDCl}_{3}\right) \delta$ $20.43,22.66,22.93,23.41,110.72,112.80,118.30,122.06,122.89,128.82,153.98$, 154.28. 

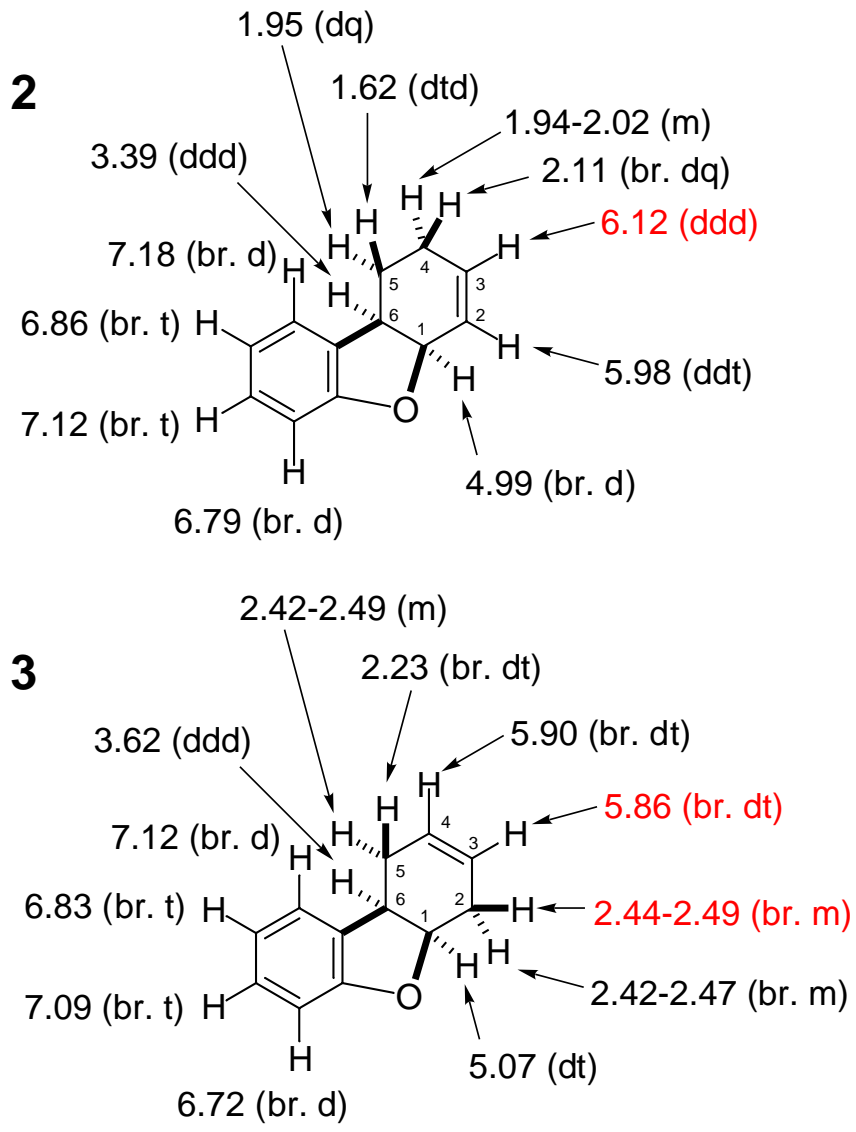

4

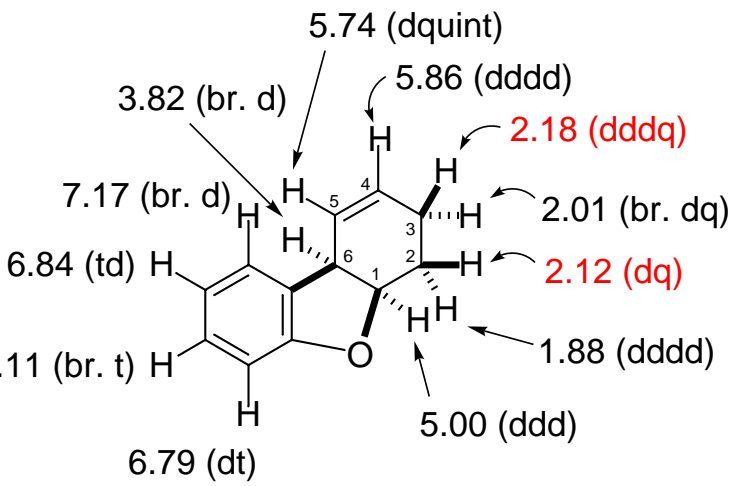

5

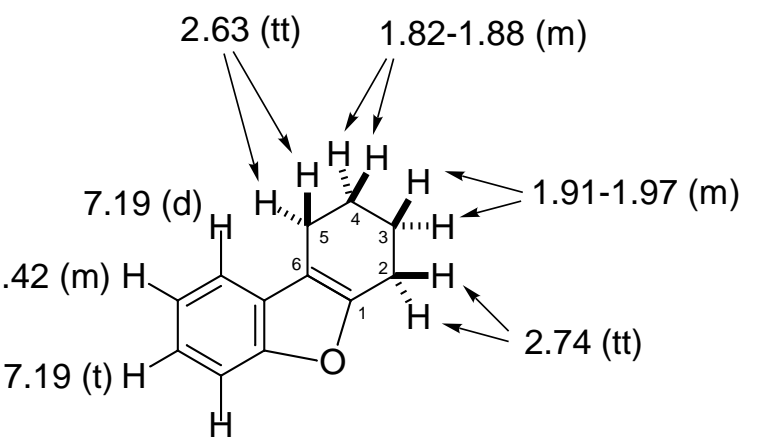

7.38-7.42 (m)

Figure 2. Chemical shifts of ${ }^{1} \mathrm{H}$ NMR for $\mathbf{2}, \mathbf{3}, \mathbf{4}$, and 5 . 
Palladium-Catalyzed Oxidative Cyclization of $c i s-3-d-1$ in the Presence of $\operatorname{Pd}(\mathrm{II}) /(\boldsymbol{S}, \boldsymbol{S})$-ip-boxax Catalyst and $\boldsymbol{p}$-Benzoquinone. ${ }^{1} \mathrm{H}$ and ${ }^{2} \mathrm{H}$ NMR spectra for the products, $\mathbf{2}$, cis-2-d-3, and cis-2-d-4 are shown in Figure 3.<smiles>[2H][C@H]1C=C[C@H](c2ccccc2O)[C@H](I)C1</smiles>

cis-3-d-1<smiles>CC1C=CC2Oc3ccccc3C2C1</smiles>

2
$\mathrm{Pd}(\mathrm{MeCN})_{4}\left(\mathrm{BF}_{4}\right)_{2}(5 \mathrm{~mol} \%)$ $(S, S)$-ip-boxax $\left(\mathrm{Pd} / \mathrm{L}^{*}=1 / 2\right)$
$78 \%$ yield

$\mathbf{2} / \mathbf{3} / \mathbf{4} / \mathbf{5}=16 / 46 / 29 / 9$ $\mathrm{MeOH}, 40^{\circ} \mathrm{C}, 4 \mathrm{~h}$

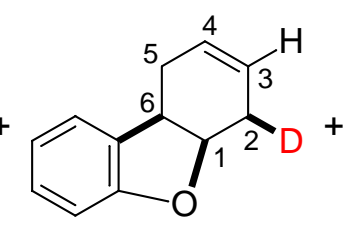

cis-2- $d-3$

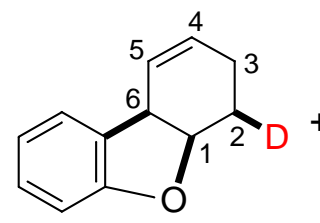

cis-2- $d-4$<smiles>[2H]C1CCCc2c1oc1ccccc21</smiles>

$2-d-5$

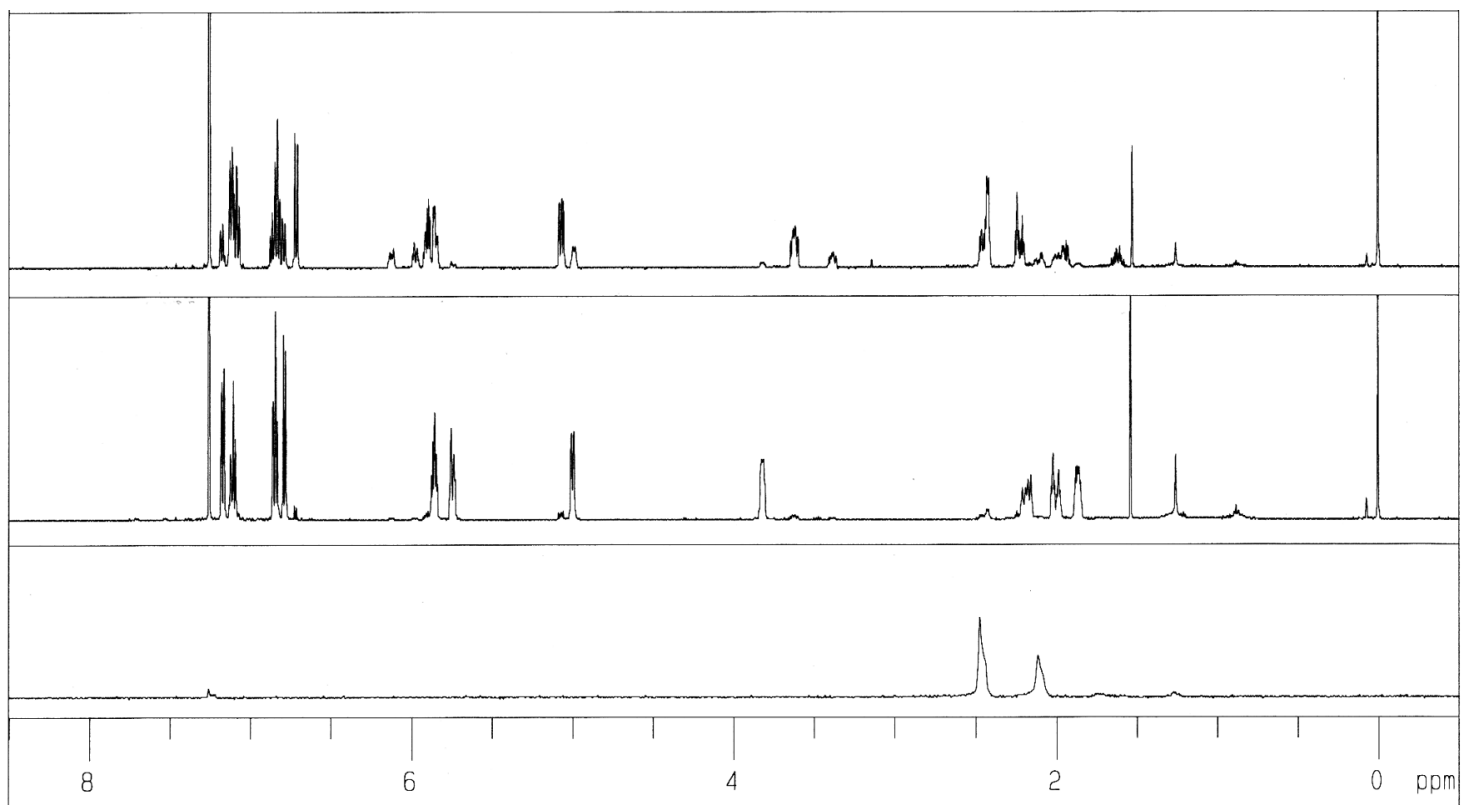

Figure 3. ${ }^{1} \mathrm{H}$ NMR chart for $2+c i s-2-d-3$ (upper), cis-2-d-4 (middle), and ${ }^{2} \mathrm{H}$ NMR chart for $\mathbf{2}+c i s-2-d-\mathbf{3}+c i s-2-d-\mathbf{4}$ (lower)

cis-2-d-3: ${ }^{1} \mathrm{H} \mathrm{NMR}\left(\mathrm{CDCl}_{3}\right) \delta 2.23($ br dt, $J=15.8,4.8 \mathrm{~Hz}, 1 \mathrm{H}), 2.43$ (br s, $\left.1 \mathrm{H}\right)$, 2.41-2.49 (m, 1H), $3.62(\mathrm{ddd}, J=9.1,6.9,5.0 \mathrm{~Hz}, 1 \mathrm{H}), 5.07(\mathrm{dt}, J=9.1,5.5 \mathrm{~Hz}, 1 \mathrm{H})$, 5.86 (br dd, $J=9.6,4.5 \mathrm{~Hz}, 1 \mathrm{H}), 5.90(\mathrm{dtd}, J=9.6,4.5,1.5 \mathrm{~Hz}, 1 \mathrm{H}), 6.72(\mathrm{br} \mathrm{d}, J=8.0$ $\mathrm{Hz}, 1 \mathrm{H}), 6.83$ (br t, $J=7.5 \mathrm{~Hz}, 1 \mathrm{H}), 7.09$ (br t, $J=8.0 \mathrm{~Hz}, 1 \mathrm{H}), 7.12$ (br d, $J=7.5 \mathrm{~Hz}$, $1 \mathrm{H}) ;{ }^{2} \mathrm{H} \mathrm{NMR}\left(\mathrm{CHCl}_{3}\right) \delta 2.48(\mathrm{~s}, 1 \mathrm{D}) . c i s-2-d-4:{ }^{1} \mathrm{H}$ NMR $\left(\mathrm{CDCl}_{3}\right) \delta 1.84-1.90(\mathrm{br} \mathrm{m}, 1 \mathrm{H})$, 
2.01 (br dt, $J=17.1,5.0 \mathrm{~Hz}, 1 \mathrm{H}), 2.18(\mathrm{ddq}, J=17.1,9.7,2.4 \mathrm{~Hz}, 1 \mathrm{H}), 3.82(\mathrm{br} \mathrm{d}, J=7.5$ $\mathrm{Hz}, 1 \mathrm{H}), 5.00(\mathrm{dd}, J=7.8,3.4 \mathrm{~Hz}, 1 \mathrm{H}), 5.74$ (dquint, $J=10.0,1.9 \mathrm{~Hz}, 1 \mathrm{H}), 5.86$ (dddd, $J$ $=10.0,5.0,3.1,1.9 \mathrm{~Hz}, 1 \mathrm{H}), 6.79(\mathrm{dt}, J=8.0,0.5 \mathrm{~Hz}, 1 \mathrm{H}), 6.84(\mathrm{td}, J=7.5,1.0 \mathrm{~Hz}, 1 \mathrm{H})$, 7.11 (br t, $J=8.0 \mathrm{~Hz}, 1 \mathrm{H}), 7.17$ (br d, $J=7.5 \mathrm{~Hz}, 1 \mathrm{H}) ;{ }^{2} \mathrm{H} \mathrm{NMR}\left(\mathrm{CHCl}_{3}\right) \delta 2.12$ (s, 1D). 2$d$-5: ${ }^{1} \mathrm{H} \mathrm{NMR}\left(\mathrm{CDCl}_{3}\right) \delta 1.85$ (quint, $\left.J=6.0 \mathrm{~Hz}, 2 \mathrm{H}\right), 1.93(\mathrm{q}, J=6.0 \mathrm{~Hz}, 2 \mathrm{H}), 2.63(\mathrm{td}, J$ $=6.1,1.9 \mathrm{~Hz}, 2 \mathrm{H}), 2.74(\mathrm{br} \mathrm{s}, 1 \mathrm{H}), 7.19(\mathrm{~d}, J=7.1 \mathrm{~Hz}, 1 \mathrm{H}), 7.19(\mathrm{t}, J=5.5 \mathrm{~Hz}, 1 \mathrm{H})$, 7.38-7.42 (m, 2H); ${ }^{2} \mathrm{H} \mathrm{NMR}\left(\mathrm{CHCl}_{3}\right) \delta 2.74(\mathrm{~s}, 1 \mathrm{D}) ;{ }^{13} \mathrm{C} \mathrm{NMR}\left(\mathrm{CDCl}_{3}\right) \delta 20.44,22.64$, 22.84, 23.09 (t, $J=19.9 \mathrm{~Hz}), 110.73,112.84,118.30,122.07,122.90,128.83,154.00$, 154.30. 


\section{Palladium-Catalyzed Oxidative Cyclization of trans-3-d-1 in the Presence}

of $\operatorname{Pd}(\mathrm{II}) /(\boldsymbol{S}, \boldsymbol{S})$-ip-boxax Catalyst and $\boldsymbol{p}$-Benzoquinone. ${ }^{1} \mathrm{H}$ and ${ }^{2} \mathrm{H}$ NMR spectra for the products, 3-d-2, 3-d-3, and rans-3-d-4 are shown in Figure 4.<smiles>[2H]C1C=CC(c2ccccc2O)CC1</smiles>

trans-3-d-1<smiles>[2H]C1=CC2CC(C)CC2Oc2ccccc21</smiles>

3-d-2
$\mathrm{Pd}(\mathrm{MeCN})_{4}\left(\mathrm{BF}_{4}\right)_{2}(5 \mathrm{~mol} \%)$

$(S, S)$-ip-boxax $\left(\mathrm{Pd} / \mathrm{L}^{*}=1 / 2\right)$

$\underset{\substack{p \text {-benzoquinone }\left(4 \text { equiv) } \\ \text { MeOH, } 20^{\circ} \mathrm{C}, 4 \mathrm{~h}\right.}}{82 \% \text { yield }} \mathbf{2 / 3 / 4 / 5 = 3 3 / 3 3 / 2 3 / 1 1}$

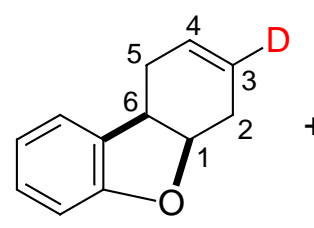

3- $d-3$

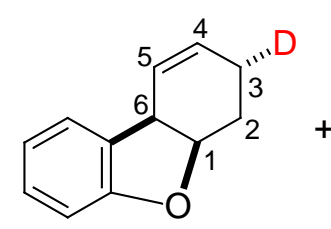

trans-3- $d-4$

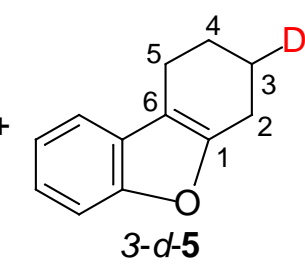

3- $d-5$

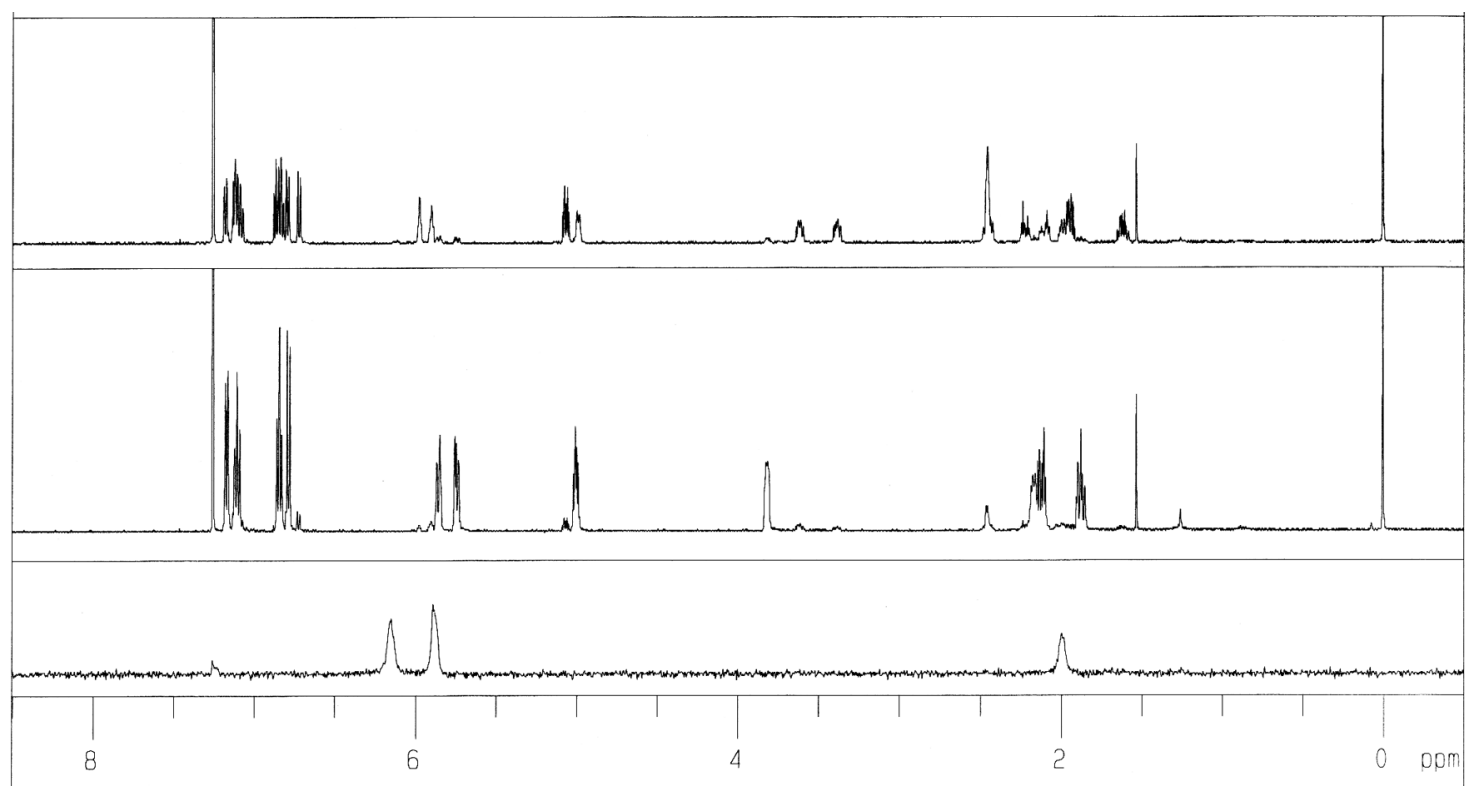

Figure 4. ${ }^{1} \mathrm{H}$ NMR chart for 3-d-2 + 3-d-3 (upper), trans-3-d-4 (middle), and ${ }^{2} \mathrm{H}$ NMR chart for 3-d-2 +3-d-3 + trans-3-d-4 (lower)

3-d-2: ${ }^{1} \mathrm{H} \mathrm{NMR}\left(\mathrm{CDCl}_{3}\right) \delta 1.62(\mathrm{dtd}, J=15.0,9.8,5.0 \mathrm{~Hz}, 1 \mathrm{H}), 1.95(\mathrm{dq}, J=15.0$, $5.0 \mathrm{~Hz}, 1 \mathrm{H}), 1.94-2.02(\mathrm{~m}, 1 \mathrm{H}), 2.10(\mathrm{br} \mathrm{dt}, J=18.0,5.0 \mathrm{~Hz}, 1 \mathrm{H}), 3.39(\mathrm{td}, J=9.8,8.3$, $4.8 \mathrm{~Hz}, 1 \mathrm{H}), 4.99$ (br d, $J=8.3 \mathrm{~Hz}, 1 \mathrm{H}), 5.98$ (br s, 1H), 6.79 (br d, $J=8.0 \mathrm{~Hz}, 1 \mathrm{H}), 6.86$ (br t, $J=7.5 \mathrm{~Hz}, 1 \mathrm{H}), 7.12$ (br t, $J=8.0 \mathrm{~Hz}, 1 \mathrm{H}), 7.18$ (br d, $J=7.5 \mathrm{~Hz}, 1 \mathrm{H}$ ); ${ }^{2} \mathrm{H}$ NMR $\left(\mathrm{CHCl}_{3}\right) \delta 6.15$ (s, 1D). 3-d-3: ${ }^{1} \mathrm{H}$ NMR $\left(\mathrm{CDCl}_{3}\right) \delta 2.23($ br dt, $J=15.9,5.1 \mathrm{~Hz}, 1 \mathrm{H})$, 
2.42-2.47 (br m, 1H), 2.42-2.49 (m, 1H), 2.44-2.49 (br m, 1H), 3.62 (ddd, $J=9.3,6.9,5.0$ $\mathrm{Hz}, 1 \mathrm{H}), 5.07$ (dt, $J=9.2,5.0 \mathrm{~Hz}, 1 \mathrm{H}), 5.90$ (br s, 1H), 6.72 (br d, $J=8.0 \mathrm{~Hz}, 1 \mathrm{H}), 6.83$ (br t, $J=7.5 \mathrm{~Hz}, 1 \mathrm{H}), 7.09$ (br t, $J=8.0 \mathrm{~Hz}, 1 \mathrm{H}), 7.12($ br d, $J=7.5 \mathrm{~Hz}, 1 \mathrm{H}) ;{ }^{2} \mathrm{H}$ NMR $\left(\mathrm{CHCl}_{3}\right) \delta 5.89$ (s, 1D). trans-3-d-4: ${ }^{1} \mathrm{H} \mathrm{NMR}\left(\mathrm{CDCl}_{3}\right) \delta 1.88(\mathrm{ddd}, J=13.7,9.7,3.4 \mathrm{~Hz}$, 1H), $2.12(\mathrm{dt}, J=13.7,5.0 \mathrm{~Hz}, 1 \mathrm{H}), 2.18(\mathrm{ddq}, J=9.7,5.0,2.4 \mathrm{~Hz}, 1 \mathrm{H}), 3.82(\mathrm{br} \mathrm{d}, J=$ $7.8 \mathrm{~Hz}, 1 \mathrm{H}), 5.00(\mathrm{ddd}, J=7.8,5.0,3.4 \mathrm{~Hz}, 1 \mathrm{H}), 5.74(\mathrm{ddd}, J=10.0,3.7,2.4 \mathrm{~Hz}, 1 \mathrm{H})$, $5.86($ br d, $J=10.0 \mathrm{~Hz}, 1 \mathrm{H}), 6.79(\mathrm{dd}, J=8.0,0.5 \mathrm{~Hz}, 1 \mathrm{H}), 6.84$ (br t, $J=7.5 \mathrm{~Hz}, 1 \mathrm{H})$, 7.11 (br t, $J=8.0 \mathrm{~Hz}, 1 \mathrm{H}), 7.17$ (br d, $J=7.5 \mathrm{~Hz}, 1 \mathrm{H}) ;{ }^{2} \mathrm{H} \mathrm{NMR}\left(\mathrm{CHCl}_{3}\right) \delta 2.00$ (s, 1D). 3d-5: ${ }^{1} \mathrm{H} \mathrm{NMR}\left(\mathrm{CDCl}_{3}\right) \delta 1.85(\mathrm{q}, J=6.1 \mathrm{~Hz}, 2 \mathrm{H}), 1.89-1.97(\mathrm{~m}, 1 \mathrm{H}), 2.63(\mathrm{tt}, J=6.1,2.0$ $\mathrm{Hz}, 2 \mathrm{H}), 2.74(\mathrm{~d}, J=6.2 \mathrm{~Hz}, 2 \mathrm{H}), 7.19(\mathrm{~d}, J=7.1 \mathrm{~Hz}, 1 \mathrm{H}), 7.19$ (t, $J=5.5 \mathrm{~Hz}, 1 \mathrm{H}), 7.38-$ $7.42(\mathrm{~m}, 2 \mathrm{H}) ;{ }^{2} \mathrm{H} \mathrm{NMR}\left(\mathrm{CHCl}_{3}\right) \delta 1.94(\mathrm{~s}, 1 \mathrm{D}) ;{ }^{13} \mathrm{C} \mathrm{NMR}\left(\mathrm{CDCl}_{3}\right) \delta 20.41,22.56,22.58(\mathrm{t}$, $J=19.6 \mathrm{~Hz}), 23.32,110.73,112.80,118.30,122.07,122.90,128.83,154.00,154.29$.

For comparison of the cyclization products obtained from 1, cis-3-d-1, and trans-3-d-1, their ${ }^{1} \mathrm{H}$ and ${ }^{2} \mathrm{H}$ NMR spectra are summarized in Figure 5. 
(a) ${ }^{1} \mathrm{H}-\mathrm{NMR}$

(b) ${ }^{1} \mathrm{H}-\mathrm{NMR}$

4

(c) ${ }^{1} \mathrm{H}-\mathrm{NMR}$

$2+3$

from cis-3-d-1

(d) 1

4

from cis-3-d-1

(e) ${ }^{2} \mathrm{H}-\mathrm{NMR}$

$2+3+4$

from cis-3-d-1

NMR

$2+3$

from trans-3- $d-1$

(g) ${ }^{1} \mathrm{H}-\mathrm{NMR}$

4

from trans-3-d-1

(h) ${ }^{2} \mathrm{H}-\mathrm{NMR}$

$2+3+4$

from trans-3- $d-1$
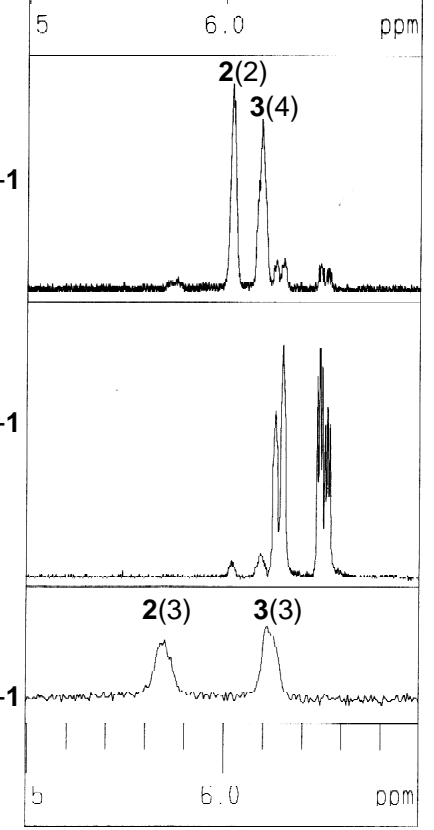
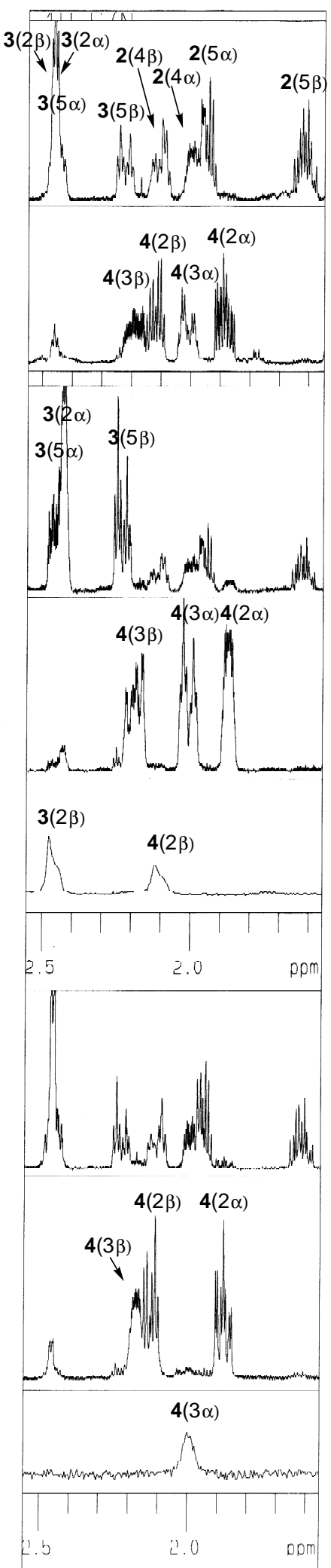

Figure 5. (a) and (b) ${ }^{1} \mathrm{H}$ NMR chart for $\mathbf{2}+\mathbf{3}$ and $\mathbf{4}$ formed from non-deuterated substrate 1. (c), (d), and (e) ${ }^{1} \mathrm{H}$ NMR chart for $2+$ cis-2-d-3, cis-2-d-4, and ${ }^{2} \mathrm{H}$ NMR chart for $2+c i s-2-d-3+c i s-2-d-4$ formed from substrate $c i s-3-d-1$. (f), (g), and (h) ${ }^{1} \mathrm{H}$ NMR chart for 3-d-2 + 3-d-3, trans-3-d-4, and ${ }^{2} \mathrm{H}$ NMR chart for 3-d-2 + 3-d-3 + trans3-d-4 formed from substrate trans-3-d-1. 
Palladium-Catalyzed Oxidative Cyclization of cis-3-d-1 in the Presence of (Pinene)Pd(II) Catalyst and Copper(II) Acetate. A mixture of $12.0 \mathrm{mg}(40 \mu \mathrm{mol})$ of bis[acetoxy(3,2,10- $\eta^{3}$-pinene)palladium](II), $7.3 \mathrm{mg}$ (40 $\left.\mu \mathrm{mol}\right)$ of copper(II) acetate, $70.0 \mathrm{mg}$ $(0.40 \mathrm{mmol})$ of $c i s-3-d \mathbf{- 1}$, and $\mathrm{MeOH}(0.8 \mathrm{~mL})$ was refluxed under oxygen for $12 \mathrm{~h}$. The mixture was concentrated in vacuo, and the residue was chromatographed on silica gel $($ EtOAc/n-hexane $=1 / 10)$ to give $56.5 \mathrm{mg}(82 \%)$ of the Wacker-type cyclization product which consists of three regioisomers 2 , cis-2-d-3, and cis-2-d-4 in a ratio of 83/14/3.<smiles>[2H][C@@H]1C=C[C@H](c2ccccc2O)[C@H](C)C1</smiles>

cis-3-d-1

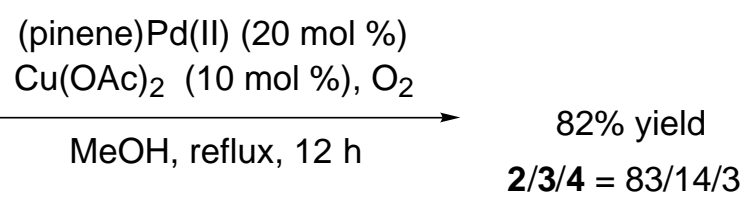

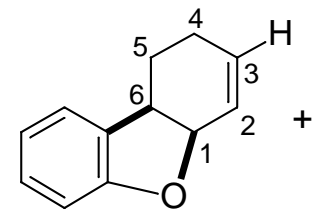

2<smiles>[OH2+]C1C([PH3+])C=CC2c3ccccc3OC21</smiles>

cis-2- $d-3$<smiles>[2H][C@@H]1CC=C[C@H]2c3ccccc3O[C@@H]21</smiles>

cis-2- $d-4$

Palladium-Catalyzed Oxidative Cyclization of cis-3-d-1 in the Presence of $\operatorname{PdCl}_{2}(\mathrm{MeCN})_{2}$, Lithium Chloride, and $\boldsymbol{p}$-Benzoquinone. A mixture of $10.4 \mathrm{mg}$ (40 $\mu \mathrm{mol})$ of dichlorobis(acetonitrile)palladium(II), $33.9 \mathrm{mg}(0.80 \mathrm{mmol})$ of lithium chloride, 84.8 $\mathrm{mg}(0.80 \mathrm{mmol})$ of sodium carbonate, $43.2 \mathrm{mg}(0.40 \mathrm{mmol})$ of $p$-benzoquinone, $70.0 \mathrm{mg}$ $(0.40 \mathrm{mmol})$ of $c i s-3-d-1$, and THF $(0.8 \mathrm{~mL})$ was refluxed for $24 \mathrm{~h}$. The mixture was concentrated in vacuo, and the residue was chromatographed on silica gel (EtOAc/n-hexane = 1/10) to give $40.5 \mathrm{mg}(59 \%)$ of the Wacker-type cyclization product which consists of three regioisomers $\mathbf{2}$, cis-2-d-3, cis-2-d-4 , and $3-d-\mathbf{5}$ in a ratio of $6 / 5 / 7 / 82$ and $20.4 \mathrm{mg}(29 \%$ recovered) of $c i s-3-d-\mathbf{1}$. 
<smiles>[2H][C@@H]1C=C[C@H](c2ccccc2O)[C@H](C)C1</smiles>

cis-3-d-1

$\mathrm{PdCl}_{2}(\mathrm{MeCN})_{2}(10 \mathrm{~mol} \%)$

$\mathrm{Na}_{2} \mathrm{CO}_{3}$ (2 equiv), $\mathrm{LiCl}$ (2 equiv)

$p$-benzoquinone (1 equiv) THF, reflux, $24 \mathrm{~h}$

$59 \%$ yield

$2 / 3 / 4 / 5=6 / 5 / 7 / 82$<smiles>C1=CC2Oc3ccccc3C2C=C1</smiles>

2<smiles>[2H][C@@H]1C=CC[C@H]2c3ccccc3O[C@H]21</smiles>
cis- $2-d-3$<smiles>[2H][C@@H]1CC=C[C@H]2c3ccccc3O[C@H]21</smiles>

cis- $2-d-4$<smiles>[2H]C1C=Cc2c(oc3ccccc23)C1</smiles> 


\section{Preparation of $o$-Allylphenols, cis-3-d-1 and trans-3-d-1}

\section{Scheme $S 1^{a}$}
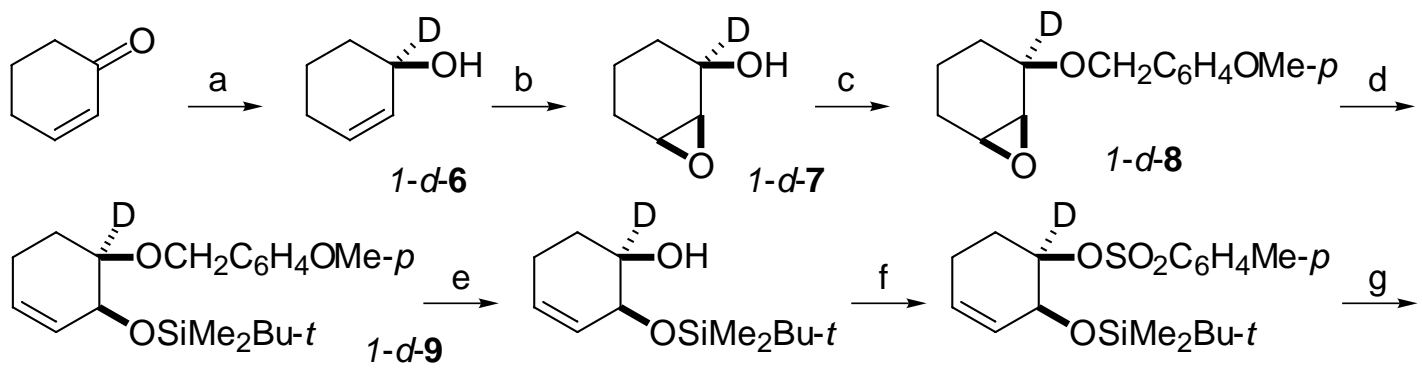

$1-d-10$<smiles>[2H]C1CCC=CC1OCCCCCCCCCC</smiles>

cis- 6- $d-12$

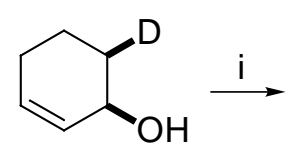

cis- $6-d-13$<smiles>[2H]C1CCC=CC1Oc1ccccc1</smiles>

cis- $6-d-14$

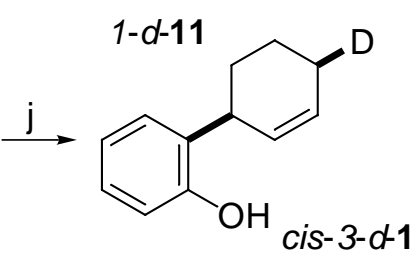

$a$ Conditions: (a) $\mathrm{LiAlD}_{4}, \mathrm{Et}_{2} \mathrm{O}, 97 \%$. (b) $\mathrm{MCPBA}, \mathrm{CH}_{2} \mathrm{Cl}_{2}, 63 \%$. (c) i) $\mathrm{NaH}$, THF. ii) $p$ - $\mathrm{MeOC}_{6} \mathrm{H}_{4} \mathrm{CH}_{2} \mathrm{Cl}$, tetrabutylammonium iodide, $83 \%$. (d) i) $t$ - $\mathrm{BuMe}_{2} \mathrm{SiCl}, 2,6$-lutidine, benzene. ii) DBU, 87\%. (e) DDQ, $\mathrm{CH}_{2} \mathrm{Cl}_{2} / \mathrm{H}_{2} \mathrm{O}, 98 \%$. (f) $p-\mathrm{MeC}_{6} \mathrm{H}_{4} \mathrm{SO}_{2} \mathrm{Cl}, \mathrm{Et}_{3} \mathrm{~N}, \mathrm{Me}_{3} \mathrm{~N} \cdot \mathrm{HCl}, \mathrm{CH}_{2} \mathrm{Cl}_{2}, 98 \%$. (g) $\mathrm{LiBEt}_{3} \mathrm{H}$, THF, 29\%. (h) tetrabutylammonium fluoride, THF, $78 \%$. (i) i) $\mathrm{NaH}$, THF. ii) $\left(\mathrm{C}_{6} \mathrm{H}_{5} \mathrm{~F}\right) \mathrm{Cr}(\mathrm{CO})_{3}$. iii) pyridine, $68 \%$. (j) $\mathrm{Eu}$ (fod) $)_{3}, \mathrm{Cl}_{2} \mathrm{CHCHCl}_{2}, 97 \%$.<smiles>OC1(O)C=CCCC1</smiles>

Preparation of 1-Deuteriocyclohex-2-en-1-ol (1-d-6). To a stirred mixture of lithium aluminum deuteride $(1.68 \mathrm{~g}, 40 \mathrm{mmol})$ in anhydrous diethyl ether $(70 \mathrm{~mL})$ at $0{ }^{\circ} \mathrm{C}$ was added dropwise a solution of 2-cyclohexenone $(7.69 \mathrm{~g}, 80 \mathrm{mmol})$ in diethyl ether $(20 \mathrm{~mL})$. The mixture was refluxed for $1 \mathrm{~h}$, diluted with diethyl ether $(10 \mathrm{~mL})$, and treated successively with water $(1.68 \mathrm{~mL}), 15 \% \mathrm{NaOH}(1.68 \mathrm{~mL})$, and water $(5.04 \mathrm{~mL})$. After stirring for $20 \mathrm{~min}$, $\mathrm{Na}_{2} \mathrm{SO}_{4}$ was added and the slurry was filtered through Celite. Evaporation of the solvent from the filtrate followed by distillation $\left(80^{\circ} \mathrm{C} / 20 \mathrm{mmHg}\right)$ gave 1-deuteriocyclohex-2-en-1-ol (7.66 g, 97\% yield). 1 - $d-6:{ }^{1} \mathrm{H}$ NMR $\left(\mathrm{CDCl}_{3}\right) \delta 1.41(\mathrm{~s}, 1 \mathrm{H}), 1.55-1.65(\mathrm{~m}, 2 \mathrm{H}), 1.69-1.76(\mathrm{~m}$, $1 \mathrm{H}), 1.84-1.91(\mathrm{~m}, 1 \mathrm{H}), 1.92-2.00(\mathrm{~m}, 1 \mathrm{H}), 2.00-2.08(\mathrm{~m}, 1 \mathrm{H}), 5.76(\mathrm{dt}, J=10.1,2.0 \mathrm{~Hz}$, $1 \mathrm{H}), 5.84(\mathrm{dt}, J=10.1,3.7 \mathrm{~Hz}, 1 \mathrm{H}) ;{ }^{2} \mathrm{H} \mathrm{NMR}\left(\mathrm{CHCl}_{3}\right) \delta 4.18(\mathrm{~s}, 1 \mathrm{D})$. 
<smiles>OC1(O)CCCC2OC21</smiles>

Preparation of cis-2,3-Epoxy-1-deuteriocyclohexan-1-ol (1-d-7). The oxidation was carried out according to the reported procedures: ${ }^{3}$ To a stirred solution of 1deuteriocyclohex-2-en-1-ol $(1-d-6)(6.43 \mathrm{~g}, 65 \mathrm{mmol})$ in dichloromethane $(50 \mathrm{~mL})$ at $0{ }^{\circ} \mathrm{C}$ was added dropwise a solution of $m$-chloroperoxybenzoic acid (MCPBA) (17.6 g, $72 \mathrm{mmol})$ in dichloromethane $(120 \mathrm{~mL})$ over a period of $1 \mathrm{~h}$, and the mixture was stirred at $0{ }^{\circ} \mathrm{C}$ for $3 \mathrm{~h}$. $\mathrm{NaHCO}_{3}(6.01 \mathrm{~g}, 72 \mathrm{mmol})$ and $\mathrm{Na}_{2} \mathrm{~S}_{2} \mathrm{O}_{3}(2.05 \mathrm{~g}, 13 \mathrm{mmol})$ were added, and the mixture was stirred for $1 \mathrm{~h}$. The precipitates were filtered off, and the filtrate was cooled to $0{ }^{\circ} \mathrm{C}$. The precipitates formed were filtered off again. Evaporation of the solvent followed by distillation $\left(150{ }^{\circ} \mathrm{C} / 3 \mathrm{mmHg}\right.$ ) gave cis-2,3-epoxy-1-deuteriocyclohexan-1-ol (8.93 g, 63\% yield). 1-d-7: ${ }^{1} \mathrm{H}$ NMR $\left(\mathrm{CDCl}_{3}\right) \delta 1.21-1.32(\mathrm{~m}, 1 \mathrm{H}), 1.41-1.50(\mathrm{~m}, 1 \mathrm{H}), 1.50-1.61(\mathrm{~m}, 2 \mathrm{H}), 1.75-1.82$ $(\mathrm{m}, 1 \mathrm{H}), 1.83-1.92(\mathrm{~m}, 1 \mathrm{H}), 1.90-2.10(\mathrm{~m}, 1 \mathrm{H}), 3.31(\mathrm{~d}, J=3.9 \mathrm{~Hz}, 1 \mathrm{H}), 3.35(\mathrm{t}, J=3.9$ $\mathrm{Hz}, 1 \mathrm{H}) ;{ }^{2} \mathrm{H} \mathrm{NMR}\left(\mathrm{CHCl}_{3}\right) \delta 4.00(\mathrm{~s}, 1 \mathrm{D})$.

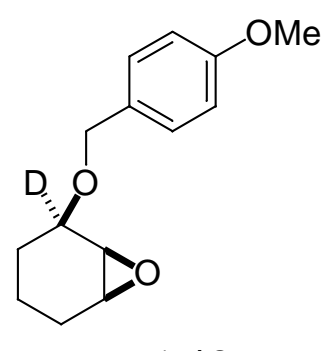

$1-d-8$

Preparation of cis-2,3-Epoxy-1-deuterio-1-(p-methoxybenzyloxy)cyclohexane (1-d-8). The MPM protection was carried out according to the reported procedures: ${ }^{4}$ To a stirred suspension of $\mathrm{NaH}(1.53 \mathrm{~g}, 64 \mathrm{mmol})$ in THF $(50 \mathrm{~mL})$ was added dropwise a solution of cis-2,3-epoxy-1-deuteriocyclohexan-1-ol (1-d-7) (6.67 g, $58 \mathrm{mmol})$ in THF (40 $\mathrm{mL})$ at $0{ }^{\circ} \mathrm{C}$. After $45 \mathrm{~min}$ at room tempetature, $p$-methoxybenzylchloride (MPMCl) $(9.99 \mathrm{~g}, 64$ mmol) and tetrabutylammonium iodide (TBAI) $(1.07 \mathrm{~g}, 2.9 \mathrm{mmol})$ were added, and the stirring was continued for $20 \mathrm{~h}$. The mixture was poured into water and extracted with ether. The extract was washed with aqueous $\mathrm{NaCl}$, dried over $\mathrm{MgSO}_{4}$, and concentrated in vacuo to leave 
an oil, which was chromatographed on a silica gel column. Elution with EtOAc/n-hexane (1/10) gave 1 - $d$-8 (11.31 g, 83\% yield) as a colorless oil. $1-d-8:{ }^{1} \mathrm{H}$ NMR $\left(\mathrm{CDCl}_{3}\right) \delta 1.16$ $1.26(\mathrm{~m}, 1 \mathrm{H}), 1.49-1.64(\mathrm{~m}, 3 \mathrm{H}), 1.76-1.87(\mathrm{~m}, 2 \mathrm{H}), 3.25(\mathrm{t}, J=3.8 \mathrm{~Hz}, 1 \mathrm{H}), 3.28(\mathrm{~d}, J=$ $3.8 \mathrm{~Hz}, 1 \mathrm{H}), 3.81(\mathrm{~d}, J=0.5 \mathrm{~Hz}, 3 \mathrm{H}), 4.615(\mathrm{~d}, J=11.9 \mathrm{~Hz}, 1 \mathrm{H}), 4.624(\mathrm{~d}, J=11.9 \mathrm{~Hz}$, $1 \mathrm{H}), 6.88(\mathrm{~d}, J=8.5 \mathrm{~Hz}, 2 \mathrm{H}), 7.31(\mathrm{~d}, J=8.3 \mathrm{~Hz}, 2 \mathrm{H}) ;{ }^{2} \mathrm{H} \mathrm{NMR}\left(\mathrm{CHCl}_{3}\right) \delta 3.77(\mathrm{~s}, 1 \mathrm{H})$; ${ }^{13} \mathrm{C} \mathrm{NMR}\left(\mathrm{CDCl}_{3}\right) \delta 19.66,23.02,24.84,53.41,54.03,55.27,69.76,73.70(\mathrm{t}, J=21.4$ $\mathrm{Hz}), 113.79$, 129.25, 130.76, 159.16. Anal. Calcd for $\mathrm{C}_{14} \mathrm{H}_{17} \mathrm{DO}_{3}$ : C, 71.46; H+D, 8.14. Found: $\mathrm{C}, 71.65 ; \mathrm{H}+\mathrm{D}, 8.00$.

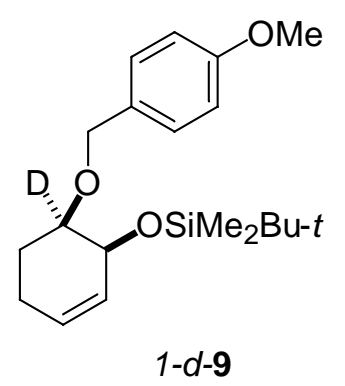

Preparation of cis-2-(t-Butyldimethylsiloxy)-1-deuterio-1-(p-methoxybenzyloxy)cyclohex-3-ene (1-d-9). The rearrangement was carried out according to the reported procedures: ${ }^{5}$ To a stirred solution of 1-d-8 (10.42 g, $\left.44 \mathrm{mmol}\right)$ and 2,6-lutidine $(23.70 \mathrm{~g}, 222 \mathrm{mmol})$ in benzene $(100 \mathrm{~mL})$ was added dropwise $t$-butyldimethylsilyl triflate $(12.86 \mathrm{~g}, 49 \mathrm{mmol})$ at $0{ }^{\circ} \mathrm{C}$. The mixture was stirred at room temperature for $30 \mathrm{~min}$ before 1,8-diazabicyclo[5.4.0]-7-undecene (DBU) (33.75 g, $222 \mathrm{mmol})$ was added, and the stirring was continued for $2 \mathrm{~h}$. The resulting mixture was poured into aqueous $\mathrm{CuSO}_{4}$ and it was extraced with ether. The exract was washed sequentially with aqueous $\mathrm{CuSO}_{4}$ and water. The organic layer was dried over $\mathrm{MgSO}_{4}$. Concentration in vacuo gave an oil, which was chromatographed on a silica gel column. Elution with EtOAc/n-hexane (1/10) gave 1-d-9 (13.45 g, 87\% yield). 1-d-9: ${ }^{1} \mathrm{H}$ NMR $\left(\mathrm{CDCl}_{3}\right) \delta 0.076(\mathrm{~s}, 3 \mathrm{H}), 0.085$ (s, 3H), 0.91 (s, 9H), 1.59-1.66 (m, 1H), 1.89-2.00 (m, 1H), 2.18-2.26 (m, 1H), $3.80(\mathrm{~s}, 3 \mathrm{H}), 4.28(\mathrm{~d}, J=$ $2.4 \mathrm{~Hz}, 1 \mathrm{H}), 4.54(\mathrm{~d}, J=12.0 \mathrm{~Hz}, 1 \mathrm{H}), 4.61(\mathrm{~d}, J=12.0 \mathrm{~Hz}, 1 \mathrm{H}), 5.61$ (ddt, $J=9.9,3.9$, $2.0 \mathrm{~Hz}, 1 \mathrm{H}), 5.74(\mathrm{dtd}, J=9.9,3.2,1.2 \mathrm{~Hz}, 1 \mathrm{H}), 6.86(\mathrm{~d}, J=8.7 \mathrm{~Hz}, 2 \mathrm{H}), 7.29(\mathrm{~d}, J=8.8$ $\mathrm{Hz}, 2 \mathrm{H}) ;{ }^{2} \mathrm{H} \mathrm{NMR}\left(\mathrm{CHCl}_{3}\right) \delta 3.51(\mathrm{~s}, 1 \mathrm{D}) ;{ }^{13} \mathrm{C} \mathrm{NMR}\left(\mathrm{CDCl}_{3}\right) \delta-4.43,-4.39,18.32,23.41$, 
23.70, 25.96, 55.26, 66.94, 70.24, 75.40 (t, $J=20.4 \mathrm{~Hz}), 113.61,128.53,129.13,129.43$, 131.39, 158.93. Anal. Calcd for $\mathrm{C}_{20} \mathrm{H}_{31} \mathrm{DO}_{3} \mathrm{Si}$ : C, 68.72; $\mathrm{H}+\mathrm{D}, 9.51$. Found: C, 68.71; $\mathrm{H}+\mathrm{D}, 9.48$.

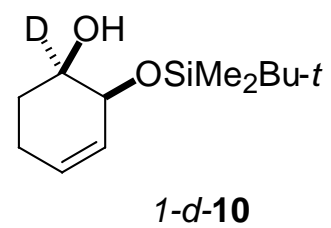

Preparation of cis-2-(t-Butyldimethylsiloxy)-1-deuteriocyclohex-3-en-1-ol (1-d-10). Deprotection of MPM ether was carried out according to the reported procedures: 6 To a stirred solution of 1-d-9 (12.35 g, $35 \mathrm{mmol})$ in dichloromethane (350 mL) containing a small amount of water (1/18 of dichloromethane) was added 2,3-dichloro-5,6-dicyano- $p$ benzoquinone (DDQ) $(8.93 \mathrm{~g}, 35 \mathrm{mmol})$ at room temperature. After stirring for $2 \mathrm{~h}$, aqueous $\mathrm{NaHCO}_{3}$ was added and the mixture was extracted with dichloromethane. The extract was washed sequentially with aqueous $\mathrm{NaHCO}_{3}$ and aqueous $\mathrm{NaCl}$, and dried over $\mathrm{MgSO}_{4}$. The solvent was evaporated and the residue was chromatographed on a silica gel column. Elution with EtOAc/n-hexane (1/10) gave $1-d-10\left(8.04 \mathrm{~g}, 98 \%\right.$ yield) as a colorless oil. $1-d-\mathbf{1 0}:{ }^{1} \mathrm{H}$ $\operatorname{NMR}\left(\mathrm{CDCl}_{3}\right) \delta 0.115(\mathrm{~s}, 3 \mathrm{H}), 0.120(\mathrm{~s}, 3 \mathrm{H}), 0.92(\mathrm{~s}, 9 \mathrm{H}), 1.67(\mathrm{dt}, J=13.0,6.5 \mathrm{~Hz}, 1 \mathrm{H})$, $1.86(\mathrm{dt}, J=13.0,6.2 \mathrm{~Hz}, 1 \mathrm{H}), 1.97(\mathrm{dm}, J=18.2 \mathrm{~Hz}, 1 \mathrm{H}), 2.22(\mathrm{dm}, J=18.1 \mathrm{~Hz}, 1 \mathrm{H})$, 2.54 (s, 1H), 4.19 (br s, 1H), 5.54 (ddt, $J=10.0,3.5,2.2 \mathrm{~Hz}, 1 \mathrm{H}), 5.81$ (dtd, $J=10.0,3.6$, $1.2 \mathrm{~Hz}, 1 \mathrm{H}) ;{ }^{2} \mathrm{H} \mathrm{NMR}\left(\mathrm{CHCl}_{3}\right) \delta 3.76(\mathrm{~s}, 1 \mathrm{D}) ;{ }^{13} \mathrm{C} \mathrm{NMR}\left(\mathrm{CDCl}_{3}\right) \delta-4.74,-4.23,18.13$, 22.47, 25.84, 26.04, 67.83 (t, $J=22.0 \mathrm{~Hz}), 67.89,127.34,130.35$. Anal. Calcd for $\mathrm{C}_{12} \mathrm{H}_{23} \mathrm{DO}_{2} \mathrm{Si}: \mathrm{C}, 62.83 ; \mathrm{H}+\mathrm{D}, 10.98$. Found: $\mathrm{C}, 62.88 ; \mathrm{H}+\mathrm{D}, 10.77$.

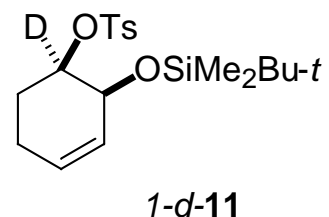

Preparation of $c i s-2-(t$-Butyldimethylsiloxy $)$-1-deuteriocyclohex-3-en-1-yl p-Toluenesulfonate (1-d-11). The tosylate formation was carried out according to the reported procedures: ${ }^{7}$ To a stirred solution of $1-d-10$ (7.98 g, $\left.35 \mathrm{mmol}\right), \mathrm{Et}_{3} \mathrm{~N}(8.84 \mathrm{~g}, 88$ 
mmol) and $\mathrm{Me}_{3} \mathrm{~N} \cdot \mathrm{HCl}(3.35 \mathrm{~g}, 35 \mathrm{mmol})$ in dichloromethane $(40 \mathrm{~mL})$ was added $p$ toluenesulfonyl chloride $(\mathrm{TsCl})(10.01 \mathrm{~g}, 53 \mathrm{mmol})$ at $0{ }^{\circ} \mathrm{C}$. The mixture was stirred at the same temperature for $4 \mathrm{~h}$. The reaction mixture was poured into water, and it was extracted with dichloromethane. The extract was washed with water, and dried over $\mathrm{MgSO}_{4}$. The solvent was evaporated and the residue was chromatographed on a silica gel column. Elution with EtOAc/n-hexane (1/10) gave 1-d-11 (13.17 g, 98\% yield) as a colorless oil. 1 - $d-11$ : ${ }^{1} \mathrm{H}$ NMR $\left(\mathrm{CDCl}_{3}\right) \delta 0.042(\mathrm{~s}, 3 \mathrm{H}), 0.044(\mathrm{~s}, 3 \mathrm{H}), 0.86(\mathrm{~s}, 9 \mathrm{H}), 1.60-1.66(\mathrm{~m}, 1 \mathrm{H}), 1.98-2.07$ (m, 1H), 2.09-2.15 (m, 1H), 2.16-2.22 (m, 1H), $2.44(\mathrm{~s}, 3 \mathrm{H}), 4.20(\mathrm{~d}, J=4.7 \mathrm{~Hz}, 1 \mathrm{H})$, 5.59 (dddd, $J=9.9,4.3,2.5,1.8 \mathrm{~Hz}, 1 \mathrm{H}), 5.72(\mathrm{dt}, J=9.9,3.4 \mathrm{~Hz}, 1 \mathrm{H}), 7.32(\mathrm{dt}, J=8.6$, $0.6 \mathrm{~Hz}, 2 \mathrm{H}), 7.81(\mathrm{dt}, J=8.6,2.0 \mathrm{~Hz}, 2 \mathrm{H}) ;{ }^{2} \mathrm{H} \mathrm{NMR}\left(\mathrm{CHCl}_{3}\right) \delta 4.60(\mathrm{~s}, 1 \mathrm{D}) ;{ }^{13} \mathrm{C} \mathrm{NMR}$ $\left(\mathrm{CDCl}_{3}\right) \delta-4.76,-4.64,18.17,21.58,22.98,24.27,25.77,65.68,80.09(\mathrm{t}, J=21.7 \mathrm{~Hz})$, 127.37, 127.67, 129.47, 129.64, 134.69, 144.34. Anal. Calcd for $\mathrm{C}_{19} \mathrm{H}_{29} \mathrm{DO}_{4} \mathrm{SSi}: \mathrm{C}$, 59.49; H+D, 8.15. Found: C, 59.79; H+D, 7.86.

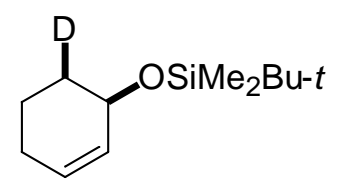

$c i s-6-d-12$

Preparation of cis-1-(t-Butyldimethylsiloxy)-6-deuteriocyclohex-2-ene (cis-6-d-12). To a stirred solution of $1-d-11$ (3.84 g, $10.0 \mathrm{mmol})$ in anhydrous THF (20 $\mathrm{mL}$ ) at $0{ }^{\circ} \mathrm{C}$ was added dropwise lithium tetraethylborohydride (Super-Hydride, $\left.\mathrm{LiBEt}_{3} \mathrm{H}\right)(20$ $\mathrm{mL}$ of $1 \mathrm{M}$ solution in THF, $20 \mathrm{mmol}$ ). The mixture was heated at reflux for $5 \mathrm{~h}$, and additional lithium tetraethylborohydride in THF $(20 \mathrm{~mL}, 20 \mathrm{mmol})$ was added. After stirring at $50{ }^{\circ} \mathrm{C}$ for 5 days, water $(0.4 \mathrm{~mL})$ was added to quench the excess hydride, and $15 \% \mathrm{NaOH}(40$ $\mathrm{ml})$ and $30 \% \mathrm{H}_{2} \mathrm{O}_{2}(40 \mathrm{~mL})$ were added to oxidize the boranes. The mixture was stirred at 40 ${ }^{\circ} \mathrm{C}$ for $2 \mathrm{~h}$ and extracted with ether. The extract was washed with water, and dried over $\mathrm{MgSO}_{4}$. The solvent was evaporated and the residue was chromatographed on a silica gel column. Elution with EtOAc/n-hexane (1/20) gave cis-6-d-12 (0.609 g, 29\% yield) as a colorless oil. cis-6-d-12: ${ }^{1} \mathrm{H}$ NMR $\left(\mathrm{CDCl}_{3}\right) \delta 0.07$ (s, 3H), 0.08 (s, 3H), 0.90 (s, 9H), 1.49- 
$1.56(\mathrm{~m}, 1 \mathrm{H}), 1.75(\mathrm{ddt}, J=13.0,7.7,5.3 \mathrm{~Hz}, 1 \mathrm{H}), 1.78-1.83(\mathrm{~m}, 1 \mathrm{H}), 1.88-1.96(\mathrm{~m}, 1 \mathrm{H})$, 1.98-2.06 (m, 1H), 4.22 (br s, 1H), 5.63 (deformed dd, $J=10.2,2.2 \mathrm{~Hz}, 1 \mathrm{H}), 5.74$ (deformed dt, $J=10.2,3.6 \mathrm{~Hz}, 1 \mathrm{H}) ;{ }^{2} \mathrm{H} \mathrm{NMR}\left(\mathrm{CHCl}_{3}\right) \delta 1.56(\mathrm{~s}, 1 \mathrm{D}) ;{ }^{13} \mathrm{C} \mathrm{NMR}\left(\mathrm{CDCl}_{3}\right) \delta$ $-4.59,-4.53,18.28,19.58,24.94,25.95,32.15(\mathrm{t}, J=19.9 \mathrm{~Hz}), 66.66,128.97,131.21$. Anal. Calcd for $\mathrm{C}_{12} \mathrm{H}_{23}$ DOSi: C, 67.54; H+D, 11.81. Found: C, 67.60; H+D, 11.61.<smiles>[2H]C1CCC=CC1O</smiles>

cis-6-d-13

Preparation of cis-6-Deuteriocyclohex-2-en-1-ol (cis-6-d-13). The deprotection of silyl ether was carried out according to the reported procedures: ${ }^{8}$ To a stirred solution of cis-6-d-12 $(0.83 \mathrm{~g}, 3.9 \mathrm{mmol})$ in anhydrous THF $(3.9 \mathrm{~mL})$ at room temperature was added tetrabutylammonium fluoride $(4.7 \mathrm{~mL}$ of $1 \mathrm{M}$ solution in THF, $4.7 \mathrm{mmol})$. After stirring for $8 \mathrm{~h}$, aqueous $\mathrm{NaCl}$ was added and the mixture was extracted with ether. The extract was washed with aqueous $\mathrm{NaCl}$, and dried over $\mathrm{MgSO}_{4}$. The solvent was evaporated and the residue was chromatographed on a silica gel column. Elution with ether/pentane (1/2) followed by distillation $\left(80{ }^{\circ} \mathrm{C} / 20 \mathrm{mmHg}\right.$ ) gave cis-6- $\mathrm{d}-\mathbf{1 3}(0.30 \mathrm{~g}, 78 \%$ yield) as a colorless oil. cis-6$d$-13: ${ }^{1} \mathrm{H} \mathrm{NMR}\left(\mathrm{CDCl}_{3}\right) \delta 1.40($ br s, $1 \mathrm{H}), 1.55-1.62(\mathrm{~m}, 1 \mathrm{H}), 1.72(\mathrm{ddt}, J=13.5,9.1,6.0$ $\mathrm{Hz}, 1 \mathrm{H}), 1.83-1.89(\mathrm{~m}, 1 \mathrm{H}), 1.97(\mathrm{dm}, J=18.2 \mathrm{~Hz}, 1 \mathrm{H}), 2.03(\mathrm{dm}, J=18.2 \mathrm{~Hz}, 1 \mathrm{H}), 4.20$ (br s, 1H), $5.76(\mathrm{ddt}, J=10.1,3.3,2.1 \mathrm{~Hz}, 1 \mathrm{H}), 5.84(\mathrm{dtd}, J=10.1,3.7,1.2 \mathrm{~Hz}, 1 \mathrm{H}) ;{ }^{2} \mathrm{H}$ $\operatorname{NMR}\left(\mathrm{CHCl}_{3}\right) \delta 1.62(\mathrm{~s}, 1 \mathrm{D}) ;{ }^{13} \mathrm{C} \mathrm{NMR}\left(\mathrm{CDCl}_{3}\right) \delta 18.79,24.95,31.52(\mathrm{t}, J=19.7 \mathrm{~Hz})$, $65.35,129.83,130.43$.<smiles>[2H]C1CCC=CC1Oc1ccccc1</smiles>

cis-6-d-14

Preparation of cis-1-Phenoxy-6-deuteriocyclohex-2-ene (cis-6-d-14). The phenyl ether formation and the removal of chromium were carried out according to the reported 
procedures: 9 To a stirred suspension of $\mathrm{NaH}(0.15 \mathrm{~g}, 3.7 \mathrm{mmol})$ in THF $(10 \mathrm{~mL})$ was added dropwise cis-6-deuteriocyclohex-2-en-1-ol (cis-6-d-13) (302 mg, $3.05 \mathrm{mmol})$ in THF (4 mL) at room temperature. The mixture was stirred for $20 \mathrm{~min}$ before fluorobenzene chromium tricarbonyl complex $\left(\mathrm{C}_{6} \mathrm{H}_{5} \mathrm{~F}\right) \mathrm{Cr}(\mathrm{CO})_{3}(1.415 \mathrm{~g}, 6.10 \mathrm{mmol})$ was added, and the stirring was continued for $4 \mathrm{~h}$. The reaction mixture was poured into aqueous $\mathrm{NaCl}$, and extracted with ether. The extract was washed with aqueous $\mathrm{NaCl}$, dried over $\mathrm{MgSO}_{4}$, and concentrated in vacuo. To the residue was added pyridine (13.8 $\mathrm{g})$, and the mixture was refluxed for $2 \mathrm{~h}$. The precipitates were filtered off, and the filtrate was diluted with ether. The mixture was washed with aqueous $\mathrm{CuSO}_{4}$ and water, and dried over $\mathrm{MgSO}_{4}$. The solvent was evaporated and the residue was chromatographed on a silica gel column. Elution with EtOAc/n-hexane (1/20) gave cis-6-d-14 (365 mg, 68\% yield) as a colorless oil. cis-6-d-14: ${ }^{1} \mathrm{H} \mathrm{NMR}\left(\mathrm{CDCl}_{3}\right) \delta 1.59-1.67$ (m, 1H), 1.810-1.88 (m, 1H), 1.90-1.95 (m, 1H), $2.03(\mathrm{dm}, J=18.3 \mathrm{~Hz}, 1 \mathrm{H}), 2.13(\mathrm{dm}, J=$ $18.3 \mathrm{~Hz}, 1 \mathrm{H}), 4.80$ (br s, 1H), 5.88 (ddt, $J=10.2,3.2,2.2 \mathrm{~Hz}, 1 \mathrm{H}), 5.97$ (dtd, $J=10.2$, 3.7, $1.2 \mathrm{~Hz}, 1 \mathrm{H}), 6.91-6.95(\mathrm{~m}, 3 \mathrm{H}), 7.27(\mathrm{t}, J=7.9 \mathrm{~Hz}, 2 \mathrm{H}) ;{ }^{2} \mathrm{H}$ NMR $\left(\mathrm{CHCl}_{3}\right) \delta 1.88(\mathrm{~s}$, 1D); ${ }^{13} \mathrm{C} \mathrm{NMR}\left(\mathrm{CDCl}_{3}\right) \delta 18.90,25.08,27.94(\mathrm{t}, J=19.9 \mathrm{~Hz}), 70.73,115.88,120.60$, 126.38, 129.46, 132.07, 157.85. Anal. Calcd for $\mathrm{C}_{12} \mathrm{H}_{13} \mathrm{DO}$ : C, 82.24; $\mathrm{H}+\mathrm{D}, 8.63$. Found: C, 82.16; H+D, 8.37.

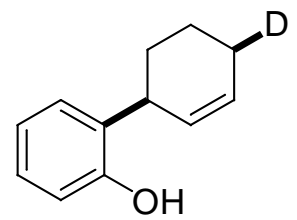

cis-3-d-1

Preparation of cis-6-(o-Hydroxyphenyl)-3-deuteriocyclohex-1-ene (cis-3$\boldsymbol{d - 1}$. The Claisen rearrangement was carried out according to the reported procedures: ${ }^{10} \mathrm{~A}$ mixture of cis-6-d-14 (227 mg, $1.30 \mathrm{mmol})$ and tris(1,1,1,2,2,3,3-heptafluoro-7,7-dmethyl4,6-octanedionato)europium(III) $\left(\mathrm{Eu}\left(\mathrm{fod}_{3}\right)\left(14 \mathrm{mg}, 1.3 \times 10^{-2} \mathrm{mmol}\right)\right.$ in $1,1,2,2-$ tetrachloroethane $(1.0 \mathrm{~mL})$ was refluxed for $2 \mathrm{~h}$. The solvent was evaporated and the residue was chromatographed on a silica gel (preparative thin-layer chromatography, EtOAc/n-hexane $(1 / 5))$ to give cis-3-d-1 (220 mg, 97\% yield). cis-3-d-1: ${ }^{1} \mathrm{H} \mathrm{NMR}\left(\mathrm{CDCl}_{3}\right) \delta 1.60-1.71(\mathrm{~m}$, 
2H), 1.77-1.84 (m, 1H), 1.97-2.06 (m, 1H), $2.11(\mathrm{br} \mathrm{s}, 1 \mathrm{H}), 3.54-3.60(\mathrm{~m}, 1 \mathrm{H}), 5.30(\mathrm{~s}$, $1 \mathrm{H}), 5.83(\mathrm{~d}, J=10.1 \mathrm{~Hz}, 1 \mathrm{H}), 6.05(\mathrm{ddd}, J=10.0,4.7,2.6 \mathrm{~Hz}, 1 \mathrm{H}), 6.80(\mathrm{dd}, J=8.4,1.2$ $\mathrm{Hz}, 1 \mathrm{H}), 6.87(\mathrm{td}, J=7.4,1.0 \mathrm{~Hz}, 1 \mathrm{H}), 7.09-7.14(\mathrm{~m}, 2 \mathrm{H}) ;{ }^{2} \mathrm{H} \mathrm{NMR}\left(\mathrm{CHCl}_{3}\right) \delta 2.13(\mathrm{~s}$, 1D); ${ }^{13} \mathrm{C} \mathrm{NMR}\left(\mathrm{CDCl}_{3}\right) \delta 21.25,24.58(\mathrm{t}, J=19.4 \mathrm{~Hz}), 29.90,37.85,116.05,120.56$, 127.46, 129.53, 129.75, 130.70, 131.04, 153.95. Anal. Calcd for $\mathrm{C}_{12} \mathrm{H}_{13} \mathrm{DO}$ : C, 82.24; $\mathrm{H}+\mathrm{D}$, 8.63. Found: $\mathrm{C}, 81.98 ; \mathrm{H}+\mathrm{D}, 8.49$.

\section{Scheme S2a}

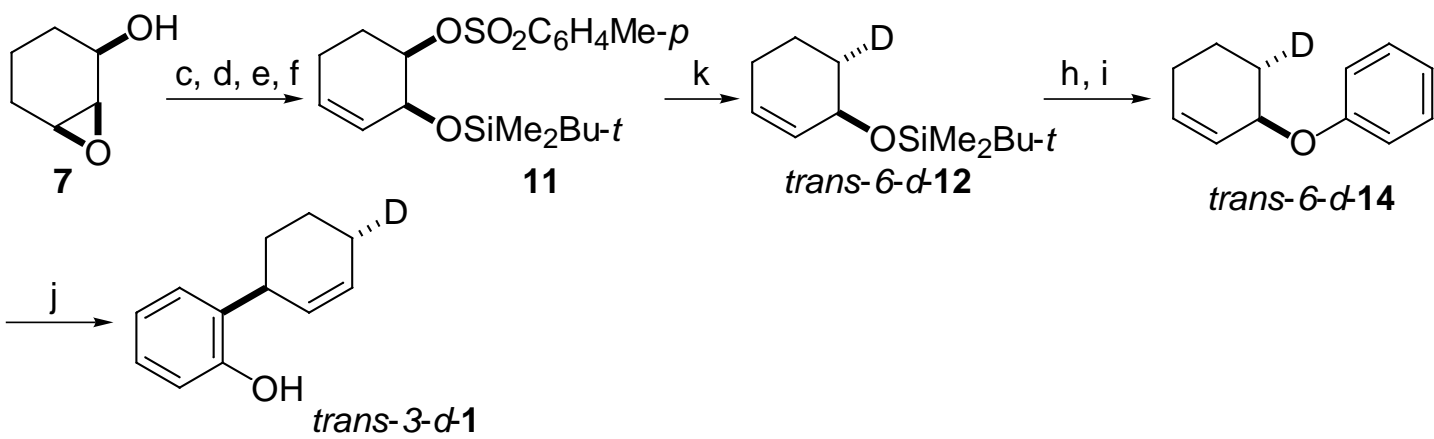

${ }^{a}$ Conditions: (c) i) $\mathrm{NaH}$, THF. ii) $p-\mathrm{MeOC}_{6} \mathrm{H}_{4} \mathrm{CH}_{2} \mathrm{Cl}$, tetrabutylammonium iodide, $83 \%$. (d) i) $t$ - $\mathrm{BuMe}_{2} \mathrm{SiCl}$, 2,6-lutidine, benzene. ii) $\mathrm{DBU}, 87 \%$. (e) $\mathrm{DDQ}, \mathrm{CH}_{2} \mathrm{Cl}_{2} / \mathrm{H}_{2} \mathrm{O}, 98 \%$. (f) $p-\mathrm{MeC}_{6} \mathrm{H}_{4} \mathrm{SO}_{2} \mathrm{Cl}$, $\mathrm{Et}_{3} \mathrm{~N}, \mathrm{Me}_{3} \mathrm{~N} \cdot \mathrm{HCl}, \mathrm{CH}_{2} \mathrm{Cl}_{2}, 98 \%$. (h) tetrabutylammonium fluoride, THF, $66 \%$. (i) i) $\mathrm{NaH}, \mathrm{THF}$. ii) $\left(\mathrm{C}_{6} \mathrm{H}_{5} \mathrm{~F}\right) \mathrm{Cr}(\mathrm{CO})_{3}$. iii) pyridine, 61\%. (j) $\mathrm{Eu}(\text { fod })_{3}, \mathrm{Cl}_{2} \mathrm{CHCHCl}_{2}, 97 \%$. (k) LiBEt 3 D, THF, $31 \%$.

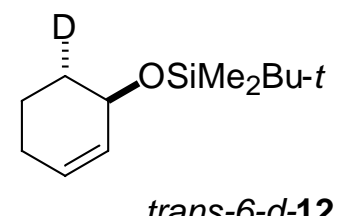

\section{Preparation of trans-1-(t-Butyldimethylsiloxy)-6-deuteriocyclohex-2-ene}

(trans-6-d-12). The tosylate 11 was prepared from epoxyalcohol 7 in essentially the same manner as the preparation of $1-d-\mathbf{1 1}$. To a stirred solution of 11 (3.83 g, $10.0 \mathrm{mmol})$ in anhydrous THF $(20 \mathrm{~mL})$ at $0{ }^{\circ} \mathrm{C}$ was added dropwise lithium tetraethylborodeuteride (SuperDeuteride, $\mathrm{LiBEt}_{3} \mathrm{D}$ ) (30 $\mathrm{mL}$ of $1 \mathrm{M}$ solution in THF, $30 \mathrm{mmol}$ ). The mixture was heated at reflux for $8 \mathrm{~h}$, and additional lithium tetraethylborohydride in THF (10 mL, $10 \mathrm{mmol}$ ) was added. The resulting mixture was heated at $50{ }^{\circ} \mathrm{C}$ for 2 days. Water $(0.4 \mathrm{~mL})$ was added to quench the excess hydride, and then $15 \% \mathrm{NaOH}(20 \mathrm{~mL})$ and $30 \% \mathrm{H}_{2} \mathrm{O}_{2}(20 \mathrm{~mL})$ were added 
to oxidize the boranes. After the mixture was stirred at $0{ }^{\circ} \mathrm{C}$ for $0.5 \mathrm{~h}$, it was extracted with ether. The extract was washed with water and dried over $\mathrm{MgSO}_{4}$. The solvent was evaporated and the residue was chromatographed on a silica gel column. Elution with EtOAc/n-hexane (1/20) gave a colorless oil of trans-6-d-12 (0.669 g, 31\% yield). trans-6-d-12: ${ }^{1} \mathrm{H}$ NMR $\left(\mathrm{CDCl}_{3}\right) \delta 0.07(\mathrm{~s}, 3 \mathrm{H}), 0.08(\mathrm{~s}, 3 \mathrm{H}), 0.90(\mathrm{~s}, 9 \mathrm{H}), 1.48-1.59(\mathrm{~m}, 2 \mathrm{H}), 1.72-1.79(\mathrm{~m}, 1 \mathrm{H})$, 1.88-1.96 (m, 1H), 1.98-2.06 (m, 1H), 4.22 (br s, 1H), 5.63 (deformed dd, $J=10.2,2.2$ $\mathrm{Hz}, 1 \mathrm{H}), 5.74$ (deformed dt, $J=10.2,3.6 \mathrm{~Hz}, 1 \mathrm{H}) ;{ }^{2} \mathrm{H} \mathrm{NMR}\left(\mathrm{CHCl}_{3}\right) \delta 1.83(\mathrm{~s}, 1 \mathrm{D}) ;{ }^{13} \mathrm{C}$ NMR $\left(\mathrm{CDCl}_{3}\right) \delta-4.59,-4.53,18.28,19.58,24.94,25.95,32.15(\mathrm{t}, J=19.9 \mathrm{~Hz}), 66.66$, 128.97, 131.21 .<smiles>[2H]C1CCC=CC1O</smiles>

trans-6- $d-13$

Preparation of trans-6-Deuteriocyclohex-2-en-1-ol (trans-6-d-13). The deprotection of silyl ether was carried out according to the reported procedures: ${ }^{8}$ To a stirred solution of trans-6-d-12 (1.19 g, $5.57 \mathrm{mmol})$ in anhydrous THF $(5.6 \mathrm{~mL})$ at room temperature was added tetrabutylammonium fluoride $(6.7 \mathrm{~mL}$ of $1 \mathrm{M}$ solution in THF, $6.7 \mathrm{mmol})$. After stirring for $5 \mathrm{~h}$, aqueous $\mathrm{NaCl}$ was added and the mixture was extracted with ether. The extract was washed with aqueous $\mathrm{NaCl}$, and dried over $\mathrm{MgSO}_{4}$. The solvent was evaporated and the residue was chromatographed on a silica gel column. Elution with ether/pentane (1/2) followed by distillation $\left(80{ }^{\circ} \mathrm{C} / 20 \mathrm{mmHg}\right)$ gave trans-6- $d-\mathbf{1 3}(0.361 \mathrm{~g}, 66 \%$ yield $)$ as a colorless oil. trans-6-d-13: ${ }^{1} \mathrm{H}$ NMR $\left(\mathrm{CDCl}_{3}\right) \delta 1.41(\mathrm{br} \mathrm{s}, 1 \mathrm{H}), 1.53-1.64(\mathrm{~m}, 2 \mathrm{H}), 1.69-1.77(\mathrm{~m}, 1 \mathrm{H})$, $1.97(\mathrm{dm}, J=16.1 \mathrm{~Hz}, 1 \mathrm{H}), 2.03(\mathrm{dm}, J=16.1 \mathrm{~Hz}, 1 \mathrm{H}), 4.19$ (br s, 1H), 5.76 (ddt, $J=$ 10.1, 3.3, $2.0 \mathrm{~Hz}, 1 \mathrm{H}), 5.84(\mathrm{dtd}, J=10.1,3.7,1.2 \mathrm{~Hz}, 1 \mathrm{H}) ;{ }^{2} \mathrm{H}$ NMR $\left(\mathrm{CHCl}_{3}\right) \delta 1.88(\mathrm{~s}$, 1D); ${ }^{13} \mathrm{C} \mathrm{NMR}\left(\mathrm{CDCl}_{3}\right) \delta 18.79,24.95,31.52(\mathrm{t}, J=19.7 \mathrm{~Hz}), 65.35,129.83,130.43$. 
<smiles>[2H][C@@H]1CCC=CC1Oc1ccccc1</smiles>

trans-6- $d-14$

Preparation of trans-1-Phenoxy-6-deuteriocyclohex-2-ene (trans-6-d-14). The phenyl ether formation and the removal of chromium were carried out according to the reported procedures: ${ }^{9}$ To a stirred suspension of $\mathrm{NaH}(0.18 \mathrm{~g}, 4.4 \mathrm{mmol})$ in THF $(10 \mathrm{~mL})$ was added dropwise trans-6-deuteriocyclohex-2-en-1-ol (trans-6-d-13) (361 mg, $3.65 \mathrm{mmol})$ in THF (7 mL) at room temperature. The mixture was stirred for 20 min before fluorobenzene chromium tricarbonyl complex $\left(\mathrm{C}_{6} \mathrm{H}_{5} \mathrm{~F}\right) \mathrm{Cr}(\mathrm{CO})_{3}(1.69 \mathrm{~g}, 7.28 \mathrm{mmol})$ was added, and the stirring was continued for $4 \mathrm{~h}$. The reaction mixture was poured into aqueous $\mathrm{NaCl}$, and extracted with ether. The extract was washed with aqueous $\mathrm{NaCl}$, dried over $\mathrm{MgSO}_{4}$, and concentrated in vacuo. To the residue was added pyridine $(16.0 \mathrm{~g})$, and the mixture was refluxed for $1 \mathrm{~h}$. The precipitates were filtered off, and the filtrate was diluted with ether. The mixture was washed with aqueous $\mathrm{CuSO}_{4}$ and water, and dried over $\mathrm{MgSO}_{4}$. The solvent was evaporated and the residue was chromatographed on a silica gel column. Elution with EtOAc/nhexane (1/20) gave trans-6-d-14 (388 mg, 61\% yield) as a colorless oil. trans-6-d-14: ${ }^{1} \mathrm{H}$ NMR $\left(\mathrm{CDCl}_{3}\right) \delta$ 1.59-1.68 (m, 1H), 1.80-1.89 (m, 2H), 1.99-2.07 (m, 1H), 2.09-2.17 (m, 1H), 4.80 (br s, 1H), 5.88 (ddt, $J=10.2,3.0,2.1 \mathrm{~Hz}, 1 \mathrm{H}), 5.97$ (dtd, $J=10.2,3.7,1.2 \mathrm{~Hz}$, $1 \mathrm{H}), 6.91-6.95(\mathrm{~m}, 3 \mathrm{H}), 7.27(\mathrm{t}, J=7.7 \mathrm{~Hz}, 2 \mathrm{H}) ;{ }^{2} \mathrm{H} \mathrm{NMR}\left(\mathrm{CHCl}_{3}\right) \delta 1.95(\mathrm{~s}, 1 \mathrm{D}) ;{ }^{13} \mathrm{C}$ NMR $\left(\mathrm{CDCl}_{3}\right) \delta 18.91,25.05,27.92(\mathrm{t}, J=19.7 \mathrm{~Hz}), 70.73,115.84,120.57,126.38$, 129.43, 132.00, 157.81. Anal. Calcd for $\mathrm{C}_{12} \mathrm{H}_{13} \mathrm{DO}$ : C, 82.24; $\mathrm{H}+\mathrm{D}, 8.63$. Found: $\mathrm{C}$, 82.20; H+D, 8.45.<smiles>[O]C1C=CC(c2ccccc2O)CC1</smiles>

trans-3-d-1 


\section{Preparation of trans-6-(o-Hydroxyphenyl)-3-deuteriocyclohex-1-ene}

(trans-3-d-1). The Claisen rearrangement was carried out according to the reported procedures:10 A mixture of trans-6-d-14 (131 mg, $0.748 \mathrm{mmol})$ and tris $(1,1,1,2,2,3,3-$ heptafluoro-7,7-dmethyl-4,6-octanedionato)europium(III) (Eu(fod) 3 ) $\left(7.7 \mathrm{mg}, 7.4 \times 10^{-3}\right.$ $\mathrm{mmol})$ in 1,1,2,2-tetrachloroethane $(0.6 \mathrm{~mL})$ was refluxed for $2 \mathrm{~h}$. The solvent was evaporated and the residue was chromatographed on a silica gel (preparative thin-layer chromatography, EtOAc/n-hexane (1/5)) to give trans-3-d-1 (127 mg, 97\% yield). trans-3-d-1: ${ }^{1} \mathrm{H}$ NMR $\left(\mathrm{CDCl}_{3}\right) \delta 1.60-1.71(\mathrm{~m}, 2 \mathrm{H}), 1.77-1.84(\mathrm{~m}, 1 \mathrm{H}), 1.97-2.05(\mathrm{~m}, 1 \mathrm{H}), 2.08-2.15(\mathrm{~m}, 1 \mathrm{H})$, 3.54-3.59 (m, 1H), $5.30(\mathrm{~s}, 1 \mathrm{H}), 5.83(\mathrm{dtd}, J=10.0,2.7,1.0 \mathrm{~Hz}, 1 \mathrm{H}), 6.05(\mathrm{dt}, J=10.0$, $2.7 \mathrm{~Hz}, 1 \mathrm{H}), 6.80(\mathrm{dd}, J=8.4,1.2 \mathrm{~Hz}, 1 \mathrm{H}), 6.87(\mathrm{td}, J=7.4,1.2 \mathrm{~Hz}, 1 \mathrm{H}), 7.09-7.13(\mathrm{~m}$, $2 \mathrm{H}) ;{ }^{2} \mathrm{H} \mathrm{NMR}\left(\mathrm{CHCl}_{3}\right) \delta 2.11(\mathrm{~s}, 1 \mathrm{D}) ;{ }^{13} \mathrm{C} \mathrm{NMR}\left(\mathrm{CDCl}_{3}\right) \delta 21.32,24.62(\mathrm{t}, J=18.9 \mathrm{~Hz})$, $29.93,37.84,116.05,120.54,127.45,129.50,129.78,130.63,131.09,154.00$.

\section{References}

(1) (a) Uozumi, Y.; Kato, K.; Hayashi, T. J. Am. Chem. Soc. 1997, 119, 5063. (b) Uozumi, Y.; Kato, K.; Hayashi, T. J. Org. Chem. 1998, 63, 5071. (c) Uozumi, Y.; Kyota, H.; Kato, K.; Ogasawara, M.; Hayashi, T. J. Org. Chem. 1999, 64, 1620.

(2) Hosokawa, T.; Uno, T.; Inui, S.; Murahashi, S.-I. J. Am. Chem. Soc. 1981, 103, 2318.

(3) Itoh, T.; Jitsukawa, K.; Kaneda, K.; Teranishi, S. J. Am. Chem. Soc. 1979, 101, 159.

(4) Marco, J. L.; Hueso-Rodríguez, J. A. Tetrahedron Lett. 1988, 29, 2456.

(5) (a) Murata, S.; Suzuki, M.; Noyori, R. J. Am. Chem. Soc. 1979, 101, 2738. (b) Murata, S.; Suzuki, M.; Noyori, R. Bull. Chem. Soc. Jpn. 1982, 55, 247. (c) Burke, S. D.; Letourneau, J. J.; Matulenko, M. A. Tetrahedron Lett. 1999, 40, 9.

(6) Horita, K.; Yoshioka, T.; Tanaka, T.; Oikawa, Y.; Yonemitsu, O. Tetrahedron 1986, 42, 3021.

(7) Yoshida, Y.; Sakakura, Y.; Aso, N.; Okada, S.; Tanabe, Y. Tetrahedron 1999, 55, 2183. 
(8) Corey, E. J.; Venkateswarlu, A. J. Am. Chem. Soc. 1972, 94, 6190.

(9) (a) Semmelhack, M. F. In Comprehansive Organometallic Chemistry II; Abel, E. W., Stone, F. G. A., Wilkinson, G., Eds.; Pergamon; New York, 1995; Vol. 12, pp 9791015. (b) Carganico, G.; Buttero, P. D.; Maiorana, S.; Riccardi, G. J. C. S., Chem. Comm. 1978, 989.

(10) Trost, B. M.; Toste, F. D. J. Am. Chem. Soc. 1998, 120, 815. 